\title{
EL DIALECTO MEXICANO DE POCHUTLA, OAXACA ${ }^{1}$
}

\section{Por Franz BoAs}

Pochutla, capital del distrito del mismo nombre del Estado de Oaxaca, está ubicada al Oeste de Tehuantepec y al Sur de Oaxaca, aproximadamente a tres leguas del Océano Pacífico. En todas las poblaciones que están al rededor de Pochutla se hablaba el Zapoteca, pero entre los vocabularios recogidos por el eminente sabio Sr. Doctor Antonio Peñafiel, quien bondadosamente me dió permiso de hacer uso de sus importantísimas colecciones, se encuentra un vocabulario como de 80 vocablos de Pochutla, los cuales muestran claramente que allí se habla el idioma náhua o mexicano. El vocabulario lo recogieron en I 888 y en ese año ya iba desapareciendo el idioma. Los datos, aunque muy imperfectos, indican que el idioma se diferencia mucho del mexicano clásico y que su fonetismo se parece al de los dialectos del Sur.

A mi modo de ver, la cuestión de la distribución antigua de los dialectos mexicanos es importantísima y creí que valía la pena visitar el pueblo y recoger todo lo que se pudiera sacar.

Llegué a Pochutla en enero de I9I2, y quedé allá hasta fines de febrero, cuando ya no se podía conseguir más de los pocos individuos que conocen parte del idioma. Son mujeres casi todas las personas que todavía se acuerdan de algunos vocablos y frases, y no hay más que una que lo pueda hablar, conociendo, como conoce, un número bastante grande de palabras y teniendo

\footnotetext{
${ }^{1}$ El estudio del dialecto de Pochutla se hizo cuando desempeñaba el cargo de Director de la Escuela Internacional de Arqueología y Etnología mexicanas. La publicación iba a hacerse en los Anales del Museo Nacional de México. A causa de las condiciones políticas y económicas del pais el manuscrito original se perdió y no es probable que siga la impresión que ya se había principiado.
}

dominio sobre las formas gramaticales. Se llama Sabina Martínez y es una anciana como de 75 años. Desgraciadamente no fué posible explicarle que para apuntar las formas se necesita una pronunciación clara y lenta y la repetición de las mismas frases. Siempre cambiaba ella la forma de las frases y por esa razón fué muy difícil recoger un buen acopio de datos. Después de unos cuantos días empezó a creer que hablar lentamente era repetir la primera sílaba del vocablo despacio y después pronunciar toda la palabra o toda la frase muy de prisa. Aunque otras mujeres, con las cuales estaba trabajando, le explicaron muchas veces lo que quería y hasta le enseñaron como se debía hablar, fué imposible lograr una buena pronunciación. Por esa razón siempre me acompañaban Mauricia Riquel, anciana muy inteligente que recuerda muchos vocablos y que me ayudó repetiendo los vocablos pronunciados por Sabina, cuando ella se acordaba de ellos. Mauricia y María Trinidad son las que tienen los mejores conocimientos del idioma, después de Sabina. Son como de 65 y 70 años de edad. Otras que conocen bastantes vocablos son: Feliciana, Francisca, Joaquina y Paula Nicha, ancianas como de 75 años, Ines Vázquez, como de 60 años y Eleuteria Avesilla, quien no tiene más de 50 años. Muy escasos son los conocimientos de Andrea Castillo, señora como de 50 años, quien siempre se interesaba en el idioma, y aunque su madre no lo hablaba, había aprendido muchos vocablos y frases.

Pocos son los hombres que recuerdan el idioma. Estanislao y Epifanio Pina, hombres como de 50 años, me comunicaron un pequeño número de vocablos; Pedro Marcelino Pastor, hombre como de 60 años, es el que tiene los mayores conocimientos, relativamente al idioma mexicano, entre los hombres. 
Otros individuos que, según lo que se dice, hablan el idioma mexicano, han sido examinados, pero no tienen conocimientos útiles. Se dijo que había un vocabulario escrito, pero es muy claro que eso se refiere al vocabulario del Sr. Dr. Peñafiel, el cual ha sido escrito por el Sr. Apolonio Rosario.

La Sra. Ines Vázquez tiene fama de saber de memoria una carta que un tal Pepe escribió en el dialecto a su madre, cuando estaba preso en Oaxaca, pero la carta estaba casi toda escrita en castellano.

-Mamá Florentina, nebá (1) ntzichuá (2) mandar las expresiones que nquet ( $\left.{ }^{3}\right)$ en la prisión con cabal salud. Titéz ( $\left.{ }^{4}\right)$ con mucho cariño a nob'lugám (5). Az xichué ( $\left.{ }^{6}\right)$ perder la esperanza que nen quicéz $\left(^{7}\right)$ de la prisión. Tixchuá $\left.{ }^{8}\right)$ contestar todo lo más pronto que puedas. Nebá (1) ntzichuá (2) unos abrazos para noyé ( $\left.{ }^{9}\right)$ Florentina.-Don Pepe.

El dialecto mexicano de Pochutla es uno del grupo de dialectos meridionales del nahua, cuyo fonetismo se diferencia mucho del nahua clásico. Ese grupo de dialectos incluye los de la América Central y la mayoría de los de Tabasco, Vera-Cruz, probablemente de Chiapas y también el dialecto de Jalisco. Sus rasgos más importantes son la ausencia de consonantes africativas, antes de otras consonantes, y la substitución de la $t$ en vez de la $t l$.

El fonetismo del dialecto de Pochutla tiene otros caracteres importantes. Hay grupos de consonantes al principio de las palabras y también grupos de más de dos consonantes, los cuales nunca se encuentran en el mexicano del Valle de México y de las regiones vecinas. También hay cambios regulares de las vocales. Casi siempre el acento está en la última y parece que la elisión de vocales y el origen de grupos de consonantes se deben en parte al cambio del acento.

Las noticias que recogí sobre la gramática de Pochutla, son muy incompletas. Sin
(1) aquí
(4) guardarás
(2) te hago
(3) estoy
(7) saldré
(5) mis hijos
(6) no haz
(8) hazme
(9) mi madre

embargo, parece que hay unas cuantas formas, tal vez más antiguas que las del mexicano clásico, como la terminación del plural en quit; terminación $u$ del posesivo, y la forma $n a$ del artículo.

El vocabulario es muỳ semejante al del mexicano clásico y se reconocen fácilmente muchos vocablos. Hay unas diferencias interesantes. El vocablo ciuat, mujer, no se encuentra, sino g'lazt, que es la forma de Pochutla para quilaztli, la diosa mexicana.

\section{Fonetismo}

\section{CONSONANTES}

\begin{tabular}{|c|c|c|c|c|c|}
\hline \multirow{2}{*}{$\begin{array}{l}\text { Labiales . } \\
\text { Dentales . }\end{array}$} & $\begin{array}{l}\mathrm{Exp} \\
(\mathrm{b})\end{array}$ & p & Continuas & Africativas & $\begin{array}{c}\text { Nasale } \\
\mathrm{m}\end{array}$ \\
\hline & (d) & & $z(c)$ & $\mathrm{tz}$ & $\mathrm{n}$ \\
\hline & - & & $\mathbf{x}$ & $\mathrm{ch}$ & - \\
\hline Paladiales . & & $c(q u)$ & $h$ & - & $\tilde{\mathrm{n}}$ \\
\hline Laterales. . & - & & 1 & 一 & - \\
\hline Semivocales & $\cdot$ & $(\mathrm{gu})(\mathrm{u}$ & & & \\
\hline & & VOCALI & ES & & \\
\hline
\end{tabular}

La $e$ y la $o$ son muy semejantes a las del castellano. Creo que no hay vocales largas en Pochutla. Las vocales terminales tienen aspiración fuerte.

En el mexicano clásico todas las explosivas son insonoras, sin aspiración, mientras que en Pochutla las explosivas iniciales siempre principian sonoras y nazarizantes. Después hay oclusión lenta del conducto nasal y se pierde el carácter sonoro. Por eso la $p$ inicial se pronuncia como la transición $m b p$, la $t$ inicial como $n d t$, la $c$ inicial (antes de $o$ y $u$ ) como ñgc. El carácter nasal es más débil en la $p$ inicial que en las otras consonantes explosivas.

$\begin{array}{ll}\text { patéc } & \text { se pronuncia mbpade'c, ancho } \\ \text { pib'luc } & \text { se pronuncia mbpib'lu'c, envolver } \\ \text { pinaua' } & \text { se pronuncia mbpinaua, tener vergüenza } \\ \text { tot } & \text { se pronuncia ndtotn, piedra } \\ \text { te } & \text { se pronuncia ndte, ¿qué? } \\ \text { caxaní } & \text { se pronuncia ñgcaxani', estâ sanando }\end{array}$

patéc se pronuncia mbpade'c, ancho pib'luc se pronuncia $m b p i b ' l u^{\prime} c$, envolver pinaua' se pronuncia mbpinaua, tener vergüenza tot se pronuncia $n d t o t n$, piedra caxaní se pronuncia ñgcaxani', estâ sanando 
La explosiva, cuando se encuentra entre dos vocales, es muy suave. La $p$, en esa posición tiene casi siempre el carácter de la $b$ castellana (continua); la $t$ también es muy suave y la articulación laringeal de la vocal precedente continúa hasta que se forme la oclusión de la dicha $t$. Por esa razón, cuando la palabra se pronuncia aprisa, la $t$ es muy semejante a la $d$, y también así la $c$ es semejante a la $g$.

tapotúc se pronuncia como ndtavodúc: está contando tequét se pronuncia como ndteguétn, hombre

Las consonantes sonoras influyen de la misma manera sobre las explosivas que las siguen y preceden.

unti' se pronuncia $u n d i^{\prime}$, borracho

Las explosivas sonoras tienen un carácter muy distinto del de las insonoras, cuando una vocal $\sin$ acento ha desaparecido entre la explosiva y otra consonante sonora. En ese caso, siempre tienen el carácter sonoro, cuya pronunciación nunca cambia:

\begin{tabular}{ccc} 
Pochutla & \multicolumn{2}{c}{ Mexicano Del VALle } \\
ug'lo'm & $<$ & ocuilin gusano \\
pig'lia' & $<$ & (piqui'?) golpear \\
b'tet & $<$ & petlatl petate \\
g'lazt & $<$ & quilazti mujer
\end{tabular}

Cuando la vocal se pierde delante de una insonora, la primera consonante explosiva sigue insonora:

$$
\text { ctze }<\text { quetza levantar }
$$

Las linguales, cuando se encuentran en posición terminal, son insonoras y requieren la oclusión linguo-paladial mientras se abre la nariz, saliendo el aire por ella. Por esa razón tienen el carácter de consonantes insonoras explosivas nasales. La $z$ terminal tiene el mismo carácter, es decir, que al terminar se abre la nariz y el aliento sale por ella.

La $\tilde{n}$ se encuentra solamente delante de las $g$ y $c$ (o $q u)$ y es la $n$ modificada por las consonantes paladiales.

La $x$ es distinta de la del mexicano. En ese dialecto se articula formando una estrecha hendedura entre el paladar y la punta de la lengua, que se aplana y se pega al primero, y con otra estrechez entre el paladar y el dorso de la lengua; así es que el sonido de la $x$ mexicana se forma de dos clases de vibraciones, las unas anteriores y semejantes a las de la sh inglesa, las otras posteriores y semejantes a las de la $j$ castellana. Un sonido de esa clase se encuentra también en el zapoteca de Oaxaca. En Pochutla, cada vez que la he oído, era igual a la $s h$ inglesa pura.

Mientras que en el mexicano moderno del Valle de México todas las consonantes terminales son insonoras, hasta las nasales y la $l$, en Pochutla las nasales terminales y la $l$ quedan sonoras.

En el dialecto de Pochutla faltan unos cuantos sonidos del mexicano clásico, que son el saltillo y la consonante hui, o sea una continua paladial que principia con resonancia de la $u$ (carácter labial) y termina con resonancia de la $i$ (carácter paladial). El fonetismo del mexicano moderno del Valle de México demuestra que, no obstante el cambio de la resonancia, el sonido corresponde a una sola consonante, porque se encuentra muchas veces en posición terminal, en la que no se permite más de una consonante. Tampoco hay la $h$, consonante continua paladial con estrechez en la región de la $c(c a, c o, c u)$, como la $h u i$, pero solamente con resonancia de la $i$ (paladial). Ya he mencionado que no hay la $t l$ del mexicano, en lugar de la cual la $t$ se encuentra siempre.

Los grupos de consonantes de Pochutla son distintos de los del Valle de México. Las africativas no se pueden poner antes de otras consonantes, así como en el dialecto de Jalisco, 1 aunque en el mexicano clásico y también en el mexicano moderno del Valle de México, hay todas las combinaciones de africativas y otras consonantes:

1 Gerónimo Thomas de Aquino, Arte, Vocabulario y Confesionario en el idioma mexicano, como se usa en el obispado de Guadalaxara, I765, pp. 5, 6.

Fr. Juan Guerra. Arte de la lengua mexicana. Guadalajara, I900, p. 9. 


$\begin{array}{cl}\text { Pochutla } & \text { Mexicano DEL VALLE } \\ \xi, z<t z & \\ u z t i^{\prime} & \text { otzti embarazada } \\ u i^{\prime} z t i & \text { uitzli espina } \\ \text { mezt } & \text { metztli luna } \\ i d^{\prime} p o z t a^{\prime} c & \text { teputzli sus espaldas } \\ \text { ozc } & \text { (uetzi) cayó } \\ x<c h & \\ \text { oco'xt } & \text { oquichtli hombre } \\ \text { oxque't } & \text { ichcatl algodón } \\ \text { noquexque'm } & \text { quechquemitl mi huipil } \\ \text { totomo'xt } & \text { totomochtli mazorca }\end{array}$

En ciertas formas gramaticales, cuando la africativa se pone delante de otra consonante, también cambia y llega a ser una continua:

moztemutu'c $<$ motztemutu'c andan buscándote
motzapi'zc $<$ motzapi'tzc te parió
tixnamig'li $<$ tichnamig'li véndeme
nixmexti'c $<$ nichmexti'c me enseñó
taxpo'l $<$ tachpol perrito

En el mexicano moderno del Valle las explosivas no se pueden poner delante de otras consonantes. Cuando se encuentran en esa posición se les da una aspiración fuerte con resonancia paladial, y los sonidos se pueden escribir $p i, t i, q u i$. Muchas veces la $c$ llega a ser una continua paladial $(h)$. En Pochutla las explosivas se encuentran delante de todas las otras consonantes.

Se permiten también grupos de consonantes al principio y al fin de las palabras, cosa imposible en el mexicano clásico, del que muchas irregularidades se expliquan. La $l$ se encuentra al principio de la palabra. Hay combinaciones de tres consonantes, pero parece que se forman solamente en palabras compuestas.

Entre el dialecto de Pochutla y el del Valle de México hay un número de cambios fonéticos regulares:

I. $\bar{a}$ (larga) del mexicano se vuelve $a$ en Pochutla:

$\begin{array}{lc}\text { Pochutla } & \text { Mexicano } \\ a y u^{\prime} t & \bar{a} y u t l \text { tortuga } \\ a p a^{\prime} z t & \bar{a} p a z t l i \text { olla } \\ a m e^{\prime} t & \bar{a} m a t l \text { papel } \\ \text { at } & \bar{a} t l \text { agua }\end{array}$

$\begin{array}{lc}\text { Pochutla } & \text { Mexicano } \\ a t o^{\prime} m t & \bar{a} t e m i t l \text { piojo } \\ a c i^{\prime} & \bar{a} c i \text { encontrar } \\ a c & \bar{a} c \text { quien } \\ a q u e^{\prime} t & \bar{a} c a t l \text { carrizo }\end{array}$

2. à del mexicano se vuèlve $a$ en Pochutla: $\begin{array}{ll}\text { taca' }^{\prime} & \text { tlàca medio dia } \\ \text { tatenli' } & \text { tlàtlani preguntar }\end{array}$

3. $a$ del mexicano se vuelve $e$ en Pochutla:

$\begin{array}{ll}\text { ame't } & \text { āmatl papel } \\ \text { etu'l } & \text { atolli atole } \\ \text { iye'c } & \text { iyac huele } \\ \text { (yeque't) } & \text { yacatl nariz } \\ \text { ozte't } & \text { iztatl sal } \\ \text { tepu' } & \text { tlapoa abrir } \\ \text { tepo'xt } & \text { tlapechtli cama } \\ \text { teque't } & \text { tlacatl hombre } \\ \text { teloa } & \text { tlaloa correr }\end{array}$

4. $e$ del mexicano, con acento, se vuelve $o$ en Pochutla:

$\begin{array}{ll}\text { ato'mt } & \text { atemitl piojo } \\ \text { pot } & \text { petlatl petate } \\ \text { mot } & \text { metlatl metate } \\ \text { tepo'xt } & \text { tlapechtli cama } \\ \text { tot } & \text { tetl piedra } \\ \text { toço' } & \text { teci }{ }^{1} \text { moler } \\ \text { toxt } & \text { textli harina } \\ \text { noxt } & \text { nextli cenizas } \\ \text { çon } & \text { centli mazorca } \\ \text { coxqui } & \text { (quequexquia) comezón } \\ \text { cocxt } & \text { quechtli pescuezo }\end{array}$

En unos cuantos vocablos se encuentran dos formas, una en $o$, otra en $e$; pero parece que la forma en $o$ es mucho más frecuente:
b'tet y pot quext y coxt
petlatl petate quechtli pescuezo

5. La $e$ y la $i$, sin acento, del mexicano corresponden a una oclusión de la glotis:

\begin{tabular}{ll} 
at'bet & altepetl pueblo \\
d'potz & teputzli espalda \\
nod'mu'z & temo voy a bajar \\
$n^{\prime} q u i$ & nequi querer \\
$z^{\prime} l i$ & celic tierno \\
$x m o c t z e^{\prime}$ & (quetza) ipárate! \\
ig'ti' & iquiti tejer \\
ptzec & pitzauac delgado \\
\multicolumn{1}{c}{ ' Según Carochi la e en teci es larga. }
\end{tabular}




$\begin{array}{ll}\text { Pochutla } & \text { Mexicano } \\ \text { enopib'lu'c } & \text { (pipiloa) me envolví } \\ \text { nob'lu' } & \text { (nopiltzin) mi hijo } \\ \text { xiub'lu' } & \text { (piloa) ¡cuélgalo! } \\ \text { mexti'c } & \text { (machitia) enseñó } \\ \text { tixnamig'li' } & \text { namiquiltia ¡véndeme! } \\ \text { xtactze } & \text { (tlaquetz) ¡habla! } \\ \text { g'lazt } & \text { quilaztli mujer }\end{array}$

6. La $i$ del mexicano se vuelve oen Pochutla:

$\begin{array}{ll}\text { ecço'c } & \text { icucic maduro } \\ \text { icoci }^{\prime} & \text { (tlanquiquici) chiflar } \\ \text { ozte't } & \text { iztatl sal } \\ \text { oxque't } & \text { ichcatl algodón } \\ \text { opque't } & \text { icpatl hilo (metatesis de la } c p \text { ) } \\ \text { oco'xt } & \text { oquichtli hombre } \\ \text { uluni' } & \text { olinia menear } \\ \text { ntapoxque'z } & \text { (pixca) voy á pizcar } \\ \text { moc } & \text { mic muerto } \\ \text { ito'c } & \text { itic adentro } \\ \text { oxt } & \text { iztetl uña } \\ \text { namocti' } & \text { namiquia casarse } \\ \text { tzucua'zt } & \text { tzicanaztli peine } \\ \text { choch } & \text { chichi escupir } \\ \text { micho'm } & \text { michin pescado }\end{array}$

Sin embargo hay muchas $i$ que no cambian:

$\begin{array}{ll}\text { aci' } & \text { aci encontrar } \\ \text { iue } & \text { icuitl hermana } \\ \text { ixqu } & \text { ixquia asar } \\ \text { ig'ti } & \text { iquiti tejer } \\ y u l i^{\prime} c & \text { yolic poco a poco } \\ u i^{\prime} t z & \text { uitz venir }\end{array}$

Casi todas las $i$ que se vuelven $o$ son breves. Parece que la $i$ larga del mexicano casi siempre es $i$ en Pochutla:

$\begin{array}{ll}i c & \bar{\imath} c \text { a donde } \\ i x t & \bar{\imath} x t l i \text { cara }\end{array}$

7. La $u i$ sin acento del mexicano corresponde a una oclusión de la glotis:

$\begin{array}{ll}\text { tag'lutu'c } & \text { (cuiloa) está escribiendo } \\ u g^{\prime} l o^{\prime} m & \text { ocuilin gusano }\end{array}$

8. La $u$ del mexicano sevuelve $o$ en Pochutla: ozc (ouetz) cayó

9. La $\bar{o}$ (larga) del mexicano se vuelve $e$ en Pochutla. Parece que ese fenómeno tiene lugar solamente en la $\bar{o}$ del preterito:

$e m o^{\prime} c$

òmic murió
Io. Las aua del mexicano se vuelven $e$ en Pochutla:

$\begin{array}{lc}\text { Pochutla } & \text { Mexicano } \\ \text { pate }^{\prime} c & \text { patlauac ancho } \\ \text { ptzec } & \text { pitzauac delgado } \\ \text { chique } c & \text { chicauac duro }\end{array}$

II. La $t l$ del mexicano se vuelve $t$ en Pochutla:

$\begin{array}{ll}\text { tzique't } & \text { tzicatl hormiga } \\ \text { xucho't } & \text { xochitl flor } \\ \text { çon } & \text { centli mazorca } \\ \text { noxt } & \text { nextli ceniza } \\ \text { neque't } & \text { nacatl carne } \\ \text { til } & \text { tlilli negro del humo } \\ \text { teque't } & \text { tlacatl hombre } \\ \text { tet } & \text { tletl fuego } \\ \text { tepo'xt } & \text { tlapachtli cama } \\ \text { teyu'l } & \text { tlayolli maiz } \\ \text { te } & \text { tle ¿qué? } \\ \text { tal } & \text { tlalli tierra } \\ \text { tayua' } & \text { tlayoa oscuro }\end{array}$

\section{ACENTO}

El acento cae casi siempre en la última sílaba.

En todas las formas que no tienen la vocal de la sílaba terminal que es característica de las formas del mexicano clásico, el acento cae en la misma sílaba en los dos dialectos:

$\begin{array}{ll}\text { apa'zt } & \text { apaztli olla } \\ \text { apoto'ct } & \text { ipotoctli humo } \\ \text { ate'n } n & \text { atentli río } \\ \text { ato'mt } & \text { atemitl piojo } \\ \text { quexque'mt } & \text { quechquemitl huipil } \\ \text { etu'l } & \text { atolli atole } \\ \text { teyu'l } & \text { tlayolli maiz }\end{array}$

Cuando la última sílaba no pierde la vocal, el acento no cae en las mismas sílabas en los dos dialectos:

$\begin{array}{ll}\text { teque't } & \text { tlacatl hombre } \\ \text { quagu't } & \text { quauitl árbol } \\ \text { micho'm } & \text { michin pescado } \\ \text { emo'c } & \text { omic murió } \\ \text { momai' } & \text { moma tu mano }\end{array}$

Hay un pequeño número de palabras cuyo acento cae en la penúltima sílaba. Parece que el acento de todas las palabras que tienen 
$e u$ en las últimas sílabas cae en la $e$. Son las formas posesivas en $e u$, como:

$$
\begin{array}{ll}
n o a t^{\prime} b e^{\prime} u & \text { mi pueblo } \\
n o g \ddot{u} e^{\prime} u & \text { mi marido }
\end{array}
$$

También:

$$
\text { cye'uc cansado }
$$

El acento de palabras que terminan en ai y oi: cae en la penúltima:

$$
\begin{array}{ll}
\text { otca' }^{\prime} i & \text { dulce } \\
\text { noxo' } i & \text { mi pié }
\end{array}
$$

Otras palabras que tienen el acento en la penúltima sílaba son:

$\begin{array}{ll}t u^{\prime} c h i & \text { pequeño } \\ t e^{\prime} i p o & \text { lagarto } \\ a^{\prime} m p a & \text { porque } \\ u i^{\prime} z t i & \text { uiztli espina }\end{array}$

El último vocablo conserva su vocal terminal. La forma de los demás sustantivos que terminan en $t l i$ en el mexicano clásico terminan en $t$ en Pochutla.

\section{GRAMÁticA}

\section{FORMAS DEL SUSTANTIVO}

En el mexicano clásico no se permiten grupos de más de dos consonantes, ni la terminación de la palabra con dos consonantes. Las raices que terminan con una consonante o con dos consonantes tienen terminaciones particulares: la mayoría de las que terminan con una consonante toman $t l i$; las que terminan con dos consonantes toman una vocal auxiliar y $t l$. En Pochutla las palabras pueden terminar con grupos de dos consonantes, y, por esa razón, no hay formas distintas de nombres en $t$ (que corresponde a la $t l$ del mexicano).

Raices que terminan con una vocal:

$\begin{array}{lc}\text { Pochutla } & \text { Mexicano } \\ \text { at } & \bar{a} t l \text { agua } \\ \text { tet } & \text { tlëtl fuego } \\ \text { tot } & \text { tetl piedra } \\ \text { cue't } & \text { coatl culebra }\end{array}$

Raices que terminan con una consonante:

$\begin{array}{lc}\text { Pochutla } & \text { Mexicano } \\ a p a^{\prime} z t & \bar{a} \text { aztli olla } \\ a p o t o^{\prime} c t & \text { ipotoctli humo } \\ \text { eyu't } & \text { ayòtli calabaza } \\ \text { ezt } & \text { eztli sangre } \\ \text { mezt } & \text { metztli luna } \\ \text { tepo'xt } & \text { tlapechtli cama } \\ \text { noxt } & \text { nextli ceniza }\end{array}$

Después de la $n$ no se oye la $t$ terminal:

$\begin{array}{ll}\text { ten } & \text { tentli boca } \\ \text { ate' } n & \text { atentli río } \\ n i x t u^{\prime} n & (\text {-tontli }) \text { pequeño } \\ \text { tzon pelo } & \end{array}$

Apolonio Rosario escribe:

$$
\begin{aligned}
& \text { adem río } \\
& \text { sont pelo }
\end{aligned}
$$

Después de la $l$ desaparece la $t$, probablemente a causa de la antigua asimilación entre la terminación $t l i$ y la $l$ :

$\begin{array}{ll}\text { etu'l } & \text { atolli atole } \\ \text { mil } & \text { milli campo } \\ \text { tal } & \text { tlalli tierra } \\ \text { teyu'l } & \text { tlayolli maiz } \\ \text { nenepi'l } & \text { nenepilli lengua }\end{array}$

Ese fenómeno es muy importante, porque demuestra que la $t$ de Pochutla procede de dos elementos fonéticos $(t \mathrm{y} t l)$. A lo menos hay la combinación de $l+t$ sin asimilación en chilto't piedra para moler $(<$ chil + tot $)$. No encontré otros ejemplos que muestren claramente si se conserva la $l$ antes de la $t$. En el dialecto de Guadalajara en el cual también falta la $t l$ hay la misma combinación, como:

$$
\text { talticpac mundo (l.c., p. I42) }
$$

Encontré una palabra que tiene la terminación $t i$ como los nombres del mexicano cuya raiz termina con una sola consonante:

$$
u i^{\prime} z t i \quad u i^{\prime} z t l i \text { espina }
$$

Las raices que terminan con dos consonantes toman una vocal auxiliar (o conservan una vocal antigua de la raiz):

$\begin{array}{ll}\text { opque't } & \text { icpatl hilo } \\ \text { ozte't } & \text { iztatl sal } \\ \text { oxque't } & \text { ichcatl algodón }\end{array}$


Es probable que en esos casos también persiste una condición más antigua, en la cual se encuentra todavía el dialecto del Valle de México.

Las raices que terminan con una $t$ tienen dos formas; las unas terminan con una vocal auxiliar, las otras no tienen terminación. La última clase es más frecuente:

$\begin{array}{lc}\text { Pochutla } & \text { Mexicano } \\ \text { b'tet y bot } & \text { petlatl petate } \\ \text { mot } & \text { metlatl metate } \\ \text { (oxt) } & \text { ixtetl uña } \\ \text { cute't } & \text { cuitlatl mierda }\end{array}$

En el mexicano clásico muchas raices que terminan con consonantes que no pueden formar el primer elemento de un grupo de consonantes tienen vocales auxiliares (o conservan vocales antiguas de la raiz). Los sonidos más importantes de esa clase son la $c$ y la $m$ (aunque las raices en $m$ tienen formas en -ntli).

Las raices que terminan en $c$ tienen también vocales auxiliares en Pochutla:

$\begin{array}{ll}\text { teque't } & \text { tlacatl hombre } \\ n e q u e^{\prime} t & \text { nacatl carne } \\ \text { ceque't } & \text { çacatl zacate } \\ \text { tzique't } & \text { tzicatl hormiga }\end{array}$

Las raices que terminan en $m$ no tienen vocal auxiliar, y corresponden a las formas clásicas en $t l i$ :

$\begin{array}{ll}\text { ato'mt }^{\prime} \text { atemitl piojo } \\ \text { quexque'mt } & \text { quechquemitl huipil } \\ \text { cumt } & \text { comitl cántaro } \\ \text { xamt } & \text { (xamitl?) tortilla }\end{array}$

Se saca:

$$
\text { ot omitl hueso }
$$

Hay una clase de nombres que terminan en om o em. Los nombres de ciertos animales pertenecen a esa clase:

$\begin{array}{ll}\begin{array}{l}\text { achiquelo'm } \\ \text { ug'lo'm }\end{array} & \text { camarón } \\ \text { tacho'm } & \text { ocuilin gusano } \\ \text { micho'm } & \text { michin perro } \\ \text { cuixo'm } & \text { iguana } \\ \text { picho'm } & \text { ? }\end{array}$

\begin{tabular}{|c|c|}
\hline Pochutla & Mexicano \\
\hline $\begin{array}{l}\text { todolem (Apo- } \\
\text { lonio Rosario) }\end{array}$ & totolin guajalote \\
\hline$o m e^{\prime} m$ & ome dos \\
\hline$q u i z c o^{\prime} m$ & (quezqui) cuantos \\
\hline $\operatorname{mixco} m(?)$ & — nixtamal \\
\hline huhio'm & (uey) grande \\
\hline$e y o^{\prime} m$ & $y e i$ tres \\
\hline$n a y o^{\prime} m$ & naui cuatro \\
\hline
\end{tabular}

Tal vez esa terminación corresponda a la in del mexicano clásico. No cabe duda que no forma parte de la raiz, porque se encuentra taxpo'l, perrito, de $t a c h o^{\prime} m$; pero es posible que corresponda al plural me.

\section{PLURAL}

No encontré formas distintas del plural en la mayoría de los nombres. Puede ser que eso se deba a que con adjectivos que expresan el plural no se usan formas distintas o a que ya se hayan olvidado las formas. En pocas palabras encontré la reduplicación:

$\begin{array}{ll}\text { Singular } & \text { Plural } \\ \text { g'lazt } & \text { quig'lazqui't mujer } \\ \text { cone't } & \text { cocone't niño } \\ \text { conebo'l } & \text { coconepo'l criatura } \\ \text { teque't } & \text { tetequetqui't hombre }\end{array}$

La terminación quit que tienen dos de las palabras que mencioné, corresponde a que del mexicano clásico y es terminación del plural del verbo que se halla frecuentemente:

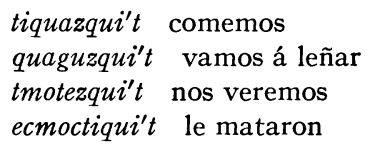

FORMAS POSESIVAS

Son muy irregulares las formas posesivas. La terminación $u h$ del mexicano corresponde a la terminación $u$ en Pochutla. A mi parecer esa era la terminación antigua del posesivo. Se pronuncia hui sonido que se ha descrito (p. I I). En Pochutla es vocal y siendo la última sílaba, como es, siempre lleva el acento. Solamente cuando la raiz termina en $e$ el acento está en la penúltima. 
Formas en $u$ con acento en la $u$ :

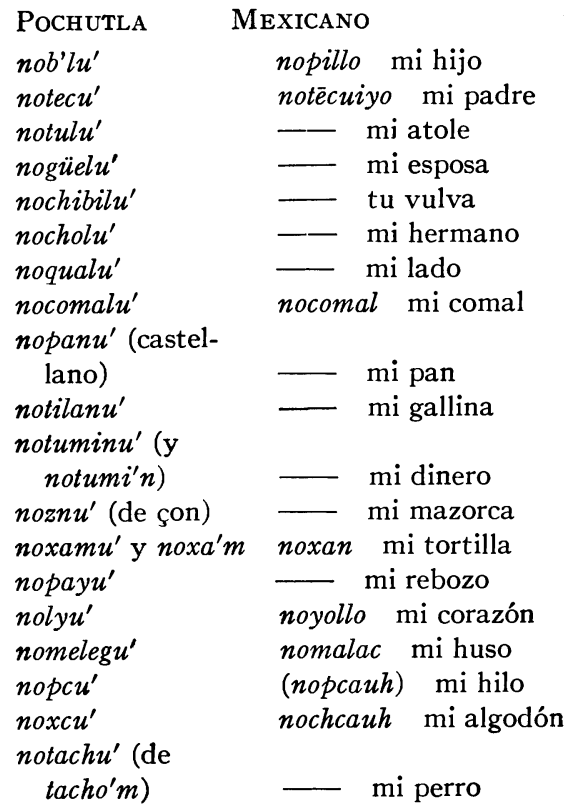

Formas en $u$ con acento en la penúltima sílaba:

\begin{tabular}{|c|c|}
\hline$\left.b e^{\prime} u\right)$ & naltpeuh mi pueblo \\
\hline nogüe'u & - mi marido \\
\hline $\begin{array}{l}\text { nocumpale } \boldsymbol{u} \\
\quad \text { (castellano) }\end{array}$ & __ mi compadre \\
\hline
\end{tabular}

Formas sin terminación:

$\begin{array}{ll}\begin{array}{l}\text { moxt } \\ \text { moi'x }\end{array} & \begin{array}{l}\text { mizte, mozte tu uña } \\ \text { mix tu cara }\end{array} \\ \text { noxca'z } & \text { mi jícara } \\ \text { noye'c } & \text { noyac mi nariz } \\ \text { ixi'c } & \text { ixic su ombligo } \\ \text { notzo'c } & \text { mi camisa } \\ \text { nomo't } & \text { nometl mi metate } \\ \text { nob'te't, nopo't } & \text { nopetl mi petate } \\ \text { mocu'm } & \text { mocon tu cántaro } \\ \text { noquexque'm } & \text { noquexquen mi huipil } \\ \text { notipe' } n & \text { nitipan mi pecho } \\ \text { note' } n & \text { noten mi boca } \\ \text { notumi'n } & \text { - mi dinero } \\ \text { notentzo'n } & \text { notentzon mi barba } \\ \text { mod'po'tz } & \text { moteputz tu espalda } \\ \text { nocha'n } n & \text { nochan mi casa } \\ \text { nob'ltzi'n } & \text { nopiltzin mi ahijado } \\ \text { notaltzi'n } & \text { notatzin mi padrino } \\ \text { nonantzi'n } & \text { nonantzin mi madrina }\end{array}$

\begin{tabular}{|c|c|}
\hline Pochutla & Mexicano \\
\hline $\begin{array}{l}\text { totoltzi' } n \\
\text { noquaxa } x t\end{array}$ & $\begin{array}{ll}\text { —_ nuestro cura } \\
\text { mi faja }\end{array}$ \\
\hline notepo'xt & (notlapech) mi cama \\
\hline $\begin{array}{l}\text { moco'ch } \\
i c h o^{\prime} c h\end{array}$ & $\begin{array}{l}\text { moquech tu pescuezo } \\
\text { ichichi su saliva }\end{array}$ \\
\hline noquanco'ch & $-\quad$ mi costal \\
\hline noneque'z & nonacaz mi oido, oreja \\
\hline nonenepill & nonenepil mi lengua \\
\hline nocel l & _ mi pene \\
\hline notupi'l & - - mi tenate \\
\hline nomil & nomil $\mathrm{mi}$ milpa \\
\hline nicu'l & $-\mathrm{mi}$ nombre \\
\hline noibe & mi hermana \\
\hline$i y e^{\prime}$ & su madre \\
\hline nopima' & mi hermana \\
\hline nomai' & noma mi mano \\
\hline noquai' & noqua mi cabeza \\
\hline nocue' $i$ & nocue $\mathrm{mi}$ enagua \\
\hline $\operatorname{noxo}^{\prime} i$ & noxo mi pié \\
\hline nixtotolu' & nixtelolo $\mathrm{mi}$ ojo \\
\hline
\end{tabular}

Segun la lista de vocablos parece que los sustantivos en tzin no tienen la terminación en $u, \mathrm{y}$ que no es frecuente en raices que terminan en $n$. Sustantivos cuyas raices terminan en una vocal no pierden la $i$ en las formas posesivas.

La contracción de la vocal del pronombre con la vocal inicial del sustantivo se encuentra cuando el sustantivo principia con la 0 :

$$
\begin{aligned}
& \text { opque't hilo nopcu' mi hilo } \\
& \text { - moxt tu uña } \\
& \text { oxque't algodón noxcu' mi algodón }
\end{aligned}
$$

Los sustantivos que principian con otras vocales no forman contracciones sino que son irregulares, encontrándose formas con contracción que son raras, y otras sin contracción que son más frecuentes:

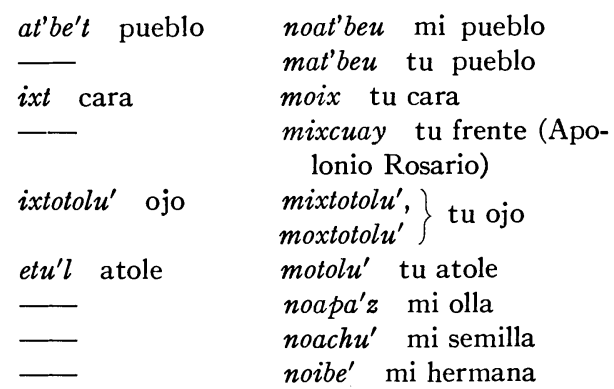


El plural de las formas posesivas tiene el sufijo gam (mex. huan). Una vez oí gan, seis veces $g a m$.

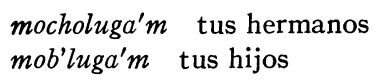

\section{COMPOSICIÓN DE LOS NOMBRES}

El diminutivo más frecuente es pol (mex. pulh).
cayupo'l caballito (de cayu')
taxpo'l perrito (de tacho'm)
g'lazpo'l muchacha (de g'lazt)
conepo'l criatura (de cone't)

El diminutivo tun (mex. ton, tontli) se encuentra solamente en

$n i x t u^{\prime} n$ pequeño

$\mathrm{El}$ reverencial es $t z i n$ (mex. $t z i n$, tzintli)

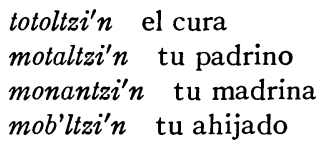

Encontré pocas postposiciones: $-c$ en

$\begin{array}{lc}\text { Pochutla } & \text { Mexicano } \\ \text { toque'lc } & \left(i t i^{\prime} c \text { calco }\right) \text { en la casa } \\ \text { ato'lc } & - \text { fuera }\end{array}$

Las otras se encuentran solamente con pronombres posesivos:

$\begin{array}{ll}\text { ito'c } & \text { itic en } \\ \text { ipe'n } & \text { itlan debajo } \\ \text { ite'nc } & \text { ixnauac frente } \\ \text { ixna'c } & \text { icpac sobre } \\ \text { tacpa'c, icpac }\end{array}$

\section{PRONOMBRES}

Los pronombres del verbo intransitivo son:

$\begin{array}{ll}\text { yo } n- & \text { nosotros } t- \\ \text { tu } t- & \text { vosotros ? } \\ \text { él }- & \text { ellos - }\end{array}$

Los pronombres del verbo reflexivo son:

$\begin{array}{ll}\text { yo-me } & \text { no-, nmo- } \\ \text { tú-te } & \text { to-? } \\ \text { él-se } & \text { mo- } \\ \text { nosotros-nos } & \text { tmo- }\end{array}$

Los pronombres del verbo transitivo son:

$\begin{array}{ll}\text { tu-me } & \text { tich- } \\ \text { él-me } & n i c h- \\ \text { yo-te } & n t z- \\ \text { él-te } & \text { motz- } \\ \text { yo-le } & n c- \\ \text { tú-le } & t i- \\ \text { él-le } & c-\end{array}$

Las formas de la segunda persona del imperativo son:

$\begin{array}{lll}\text { Verbo intransitivo } & \text { tú } & x- \\ \text { Verbo reflexivo } & \text { tú } & x o-, x m o- \\ \text { Verbo transitivo } & \text { tú-me } & \text { tich- } \\ & \text { tú-le } & x-\end{array}$

Es un rasgo característico del dialecto de Pochutla, que, con los pronombres transitivos no se usan los sujetos del intransitivo, sino que formas compuestas indican la combinación particular del sujeto y del régimen pronominal. Combinaciones de esta clase se encuentran en muchos idiomas americanos y, por esa razón, no es de suponerse que las formas de Pochutla se desarrollaron por contracción recente.

$$
\begin{array}{ll}
\text { tich }<t+\text { nech } & \text { ntz }<n+\text { mitz } \\
\text { nich }<\text { nech } & \text { motz }<\text { mitz }
\end{array}
$$

En el verbo reflexivo tampoco se usan los sujetos del verbo intransitivo, sino sujetos particulares del verbo reflexivo. Sin embargo, lo mismo que en el mexicano clásico, hay otras formas del verbo reflexivo las cuales tienen el prefijo mo con los sujetos del verbo intransitivo.

Entre el pronombre y el verbo se encuentran ligaduras que no se explican facilmente. En muchos verbos no hay ligadura; en otros se explica la ligadura por el fonetismo del dialecto, pero es claro que hay otras causas que la determinen.

Se pueden dar las reglas siguientes:

La $t$ de la segunda persona toma una ligadura antes de las consonantes dentales y paladiales. Casi siempre la ligadura es $i$.
(tamota') tirar
titamote'c tiraste
tali' poner

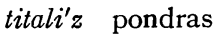
temoa' buscar
titemoa' buscas
(tuca') sembrar 


\begin{tabular}{|c|c|}
\hline namocti' & tinamocti' \\
\hline nutza' llamar & tinutza' llamas \\
\hline nqui querer & tinqui' quieres \\
\hline$($ tzecue $)$ cerrar & titzecue' cierras \\
\hline$\left(t z o m a^{\prime}\right)$ & titzome'z \\
\hline chua' hacer & tichue' haces \\
\hline$q u i c ̧ a^{\prime}$ salir & tiquiça' \\
\hline$q u a^{\prime}$ comer & $t_{i q u a^{\prime} z}$ \\
\hline comprar & ticua'z \\
\hline
\end{tabular}

La $x$ del imperativo toma una ligadura antes de las consonantes dentales, continuas y aspiradas, y antes de las paladiales. Casi siempre la ligadura es $i$.

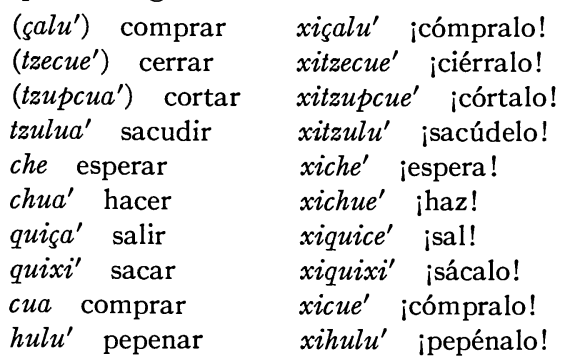

En otros verbos no se puede dar suficiente explicación.

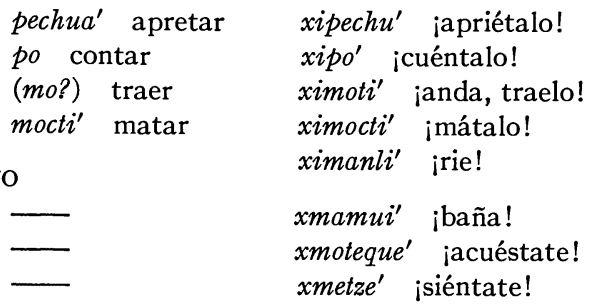

La $c$, régimen de la tercera persona, nunca toma la ligadura $i$, sino $o$. Las demás formas transitivas excepto $t$ y $x$ toman la misma ligadura. No se pueden dar reglas que indiquen cuando se toma la $o$. Los verbos que toman la $o$ toman la ligadura $i$ en la segunda persona del indicativo y del imperativo, y en la primera del plural.

\begin{tabular}{|c|c|c|}
\hline$b i a^{\prime}$ tener & ncobia' lo tengo & $\begin{array}{l}\text { tibia' lo tienes } \\
\text { tibialu't tenemos }\end{array}$ \\
\hline$p u a^{\prime}$ limpiar & encopu'c lo limpie' & xipue ilímpialo! \\
\hline$m a$ tomar & $\begin{array}{l}\text { motzuma le tomo } \\
\text { nichuma'c me tomó }\end{array}$ & xima’ ¡tómalo! \\
\hline$m a m a^{\prime}$ cargar & $\longrightarrow$ & $\begin{array}{l}\text { timama'c lo car- } \\
\text { gaste }\end{array}$ \\
\hline tepoa' abrir & $n c o t e p o^{\prime} c$ lo abrí & xitepu' ¡ábrelo! \\
\hline tuca' sembrar & $\begin{array}{l}\text { encotuque'c lo } \\
\text { sembré }\end{array}$ & $\begin{array}{l}\text { etitutque'c sem- } \\
\text { braste }\end{array}$ \\
\hline
\end{tabular}

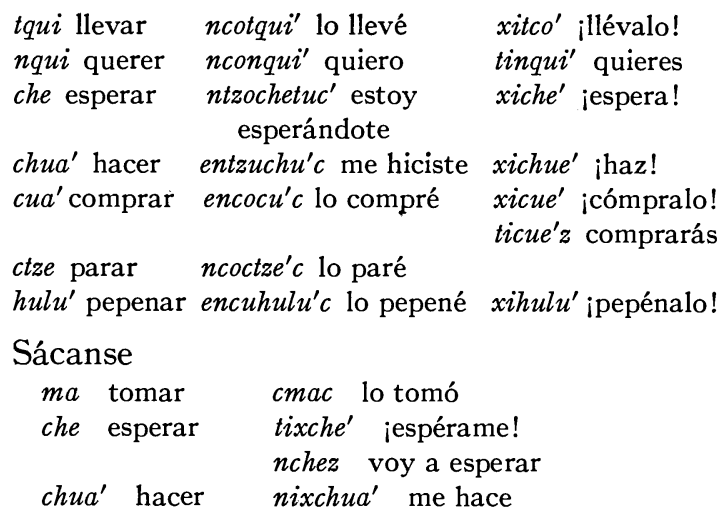

Dos verbos cuyas raices principian probablemente con $i$ toman la $o$ en la misma posición.

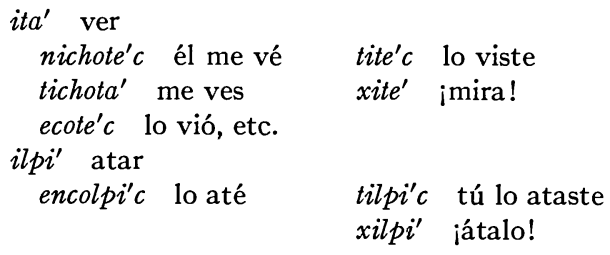

El verbo $m e c a^{\prime}$, dar siempre toma la $i$.

$$
\begin{aligned}
& \text { ntzimeca' te doy } \\
& \text { tichimeque' ¡dame! } \\
& \text { motzimequi't te dieron } \\
& \text { nichime'c él me dió } \\
& \text { timeca' das } \\
& \text { ximeque' ¡dáselo! }
\end{aligned}
$$

No estoy seguro si es de la misma clase

$$
\begin{aligned}
& \text { coxqui' comezón } \\
& \text { nicoxqui' tengo comezón }
\end{aligned}
$$

Un número pequeño de verbos tiene la ligadura $a$.

$\left(p e c a^{\prime}\right)$ lavar encape'c lo lavé xapeque' ¡lávalo!

(pelua') lamer encapelu'c lo lamí xapelu' ¡lámelo!

(pitza') parir motzapi'zc te parió (pero xipitze' isopla!)

pig'li' golpear capig'li'c lo golpeó napig'le'z voy á golpear ntzapig'le'z voy á golpearte tichapig'li' igolpéame! xapig'li' ipégalo!

$\left(q u e u a^{\prime}\right)$ guardar caqueue' $z$ ¡ve a guardarlo!

$\left(q u a n a^{\prime}\right)$ rascar xaquane' ¡ráscalo! 
Excepto las formas en $a$ que acabo de mencionar, la $n$ de la primera persona nunca toma ligadura.

Hay un número de verbos que tienen la ligadura $o$ en la segunda persona. Parece que todos son intransitivos, pero no es clara la causa que produce la $o$. No creo que sea indicación de una forma reflexiva.

$\begin{array}{ll}\text { tochuca' tú lloras } & \text { totactze' }^{\prime} \text { hablas } \\ \text { toquequi' tú oyes } & \text { toeueta'c estás viejo }^{\prime} \text { e } \\ \text { totaqua'c tú comiste } & \text { tounti' estás borracho }\end{array}$

Ejemplos de formas sin ligadura son:

\begin{tabular}{|c|c|}
\hline se mojó & $n a u e^{\prime} c$ me mojé \\
\hline$a p e^{\prime} c$ entró & napeco'z entraré \\
\hline$a c i^{\prime}$ encontrar & tichaci' me encuentras \\
\hline$i t i^{\prime}$ decir & $n^{\prime} z e t{ }^{\prime} c \quad$ le dije \\
\hline ixmeti $i^{\prime}$ conocer & nquixmeti' lo conozco \\
\hline$(y a)$ ir & niaz iré \\
\hline & $t i a^{\prime} z$ irás \\
\hline$\left(p^{\prime} n o a^{\prime}\right)$ pasar & epenu'c pasaste \\
\hline petebi' ayudar & mozpetebi'c te ayudó \\
\hline 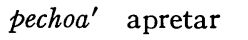 & tixpechu' ¡apriétame! \\
\hline$m a$ tomar & cmac lo tomó \\
\hline meti $i^{\prime}$ saber & cmeti' lo sé \\
\hline mexti' enseñar & $\begin{array}{l}\text { nmexti'z enseñaré } \\
\text { nixmexti'c me enseñó }\end{array}$ \\
\hline metza' sentarse & $x m e t z e^{\prime} \quad$ ¡siéntate! \\
\hline mocti $i^{\prime}$ matar & ecmoctiqui't lo mataron \\
\hline mamui' bañar & $x m a n u i^{\prime} \quad$ ¡baña! \\
\hline tapeca' lavar & ntapeque'z lavaré \\
\hline tamota' tirar & xtamote ¡tírelo! \\
\hline & enctamote'c lo tiré \\
\hline tatenli ${ }^{\prime}$ preguntar & $\begin{array}{l}\text { ntatenli'z preguntaré } \\
\text { xtatenli ipregúntale! }\end{array}$ \\
\hline tati' quemar & xtati' ¡quémalo! \\
\hline tacui'c pagó & enixtacui'c me pagó \\
\hline$m i^{\prime}$ acabar & $\begin{array}{l}\text { enctemi'c lo acabé } \\
\text { xtemi } i^{\prime} \text { ¡acábalo! }\end{array}$ \\
\hline tecu' subir & $n t e c u^{\prime} z$ subiré \\
\hline & xtecu' ¡sube! \\
\hline namig'li' vender & tixnamig'li' ivéndeme! \\
\hline amoct casarse & encnamocti'c lo casé \\
\hline tzaua' hilar & $n t z a u e^{\prime} z \quad$ hilaré \\
\hline tzupini' picar & niztzupini'c me picó \\
\hline tzulu' sacudir & entzulu'c sacudí \\
\hline che esperar & nchez voy á esperar \\
\hline chua' hacer & nixchua' me hace \\
\hline chuca' llorar & nchuca' lloro \\
\hline qua comer & nquaz comeré \\
\hline guala'c venir & nola'c vine \\
\hline
\end{tabular}

Ejemplos de los pronombres reflexivos y transitivos siguen:

Reflexivos; primera forma:

enopib'lu'c me envolví xopib'lu' ¡envuélvete!

nopina' tengo frio

$x$ otepu' ¡ábrete!

nocyeui' estoy cansado

noçute'c tengo miedo -

noxixe'z voy a mear

nococoa' estoy enfermo

enoya'c me escondí

xucochoti ivete a dormir!

$n o d^{\prime} m u^{\prime} z$ voy a bajarme $x o d^{\prime} m u^{\prime}$ ¡bájate!

Reflexivos; segunda forma:

enmoteque'c me acosté enmohue'c nen me bañé

nmoçute' me espanté

enmoco'zc me levanté

xmoteque' ¡acuéstate!

$x$ moctze' ¡levántate!

xmoyane' ¡escóndete!

Transitivos:

tú-me

tichimeque' ¡dámelo! tixpechu' ¡apriétame!

ticheti'c dijiste tixpetebi' ¡ayúdame!

ticholmeque' ¡pásamelo! tixnamig'li' ¡véndemelo!

tichapig'li' ipégame tixche' '¡espérame!

un golpe!

él-me

nichuma'c me cogió nixmexti'c me enseñó

nichime'c me lo dió nixmocti'c me mató

nichimequi't me dieron enixtacui'c ya me pagó

nichota' me mira nixtzupini'c me picó

nicheti'c me dijo

yo-te

ntzaci' te encuentro ntzimeca' te doy

ntzapig'le'z voy á pegar ntzoche' te espero

te un golpe $n t z e t i^{\prime} c$ te dije

él一te

motzapizc te parió moztemutu'c están

motzuma' te coge buscándote

mozmexti' te enseñó motzeti'c te dijo

motzimequi't te dieron

yo-lo

ncobia' lo tengo encapelu'c lo lamé

ncotepo'c lo abrí enctemi'c lo acabé

ncotqui' lo llevé enctamote'c lo tiré

encape'c lo lavé nconqui' lo quiero

tú-lo

timama'c lo cargaste timeca' se lo das

tinqui' lo quieres tichua' lo haces

ticue'z comprarás tiqua'z lo comes

tite $^{\prime} c$ lo viste 
él-lo ecote'c lo vió caqueue'z va a guardarlo ecmoctiqui't lo mataron

\section{EL PLURAL DEL VERBO}

Ya se han mencionado los plurales en quit (p. I5).

Hay otra forma que se encuentra solamente en la primera persona del plural. Me parece muy probable que esa sea la forma impersonal que tiene el sufijo lo en el mexicano clásico.

tibialu't tenemos
tuilu't nos vamos
tuitzelu't venimos
untilu't estámos borrachos
tichulu't haremos

FORMACIÓN DEL PRETÉRITO

Los verbos del mexicano clásico que terminan en el pretérito con el sonido final de la raiz, añaden en el dialecto de Pochutla $c$ a la raiz.

$\begin{array}{ll} & \text { Pochutla Y MExicANo } \\ \text { Raiz } & \text { Pretérito } \\ \text { pitz- } & \text { pizc (pitz) nacer } \\ \text { ma } & \text { mac (ma) tomar } \\ \text { mama' } & \text { mama'c (mama) cargar } \\ \text { tom- } & \text { tomc (ton) desatar } \\ \text { quiz- } & \text { quizc (quiz) salir } \\ \text { cotz- } & \text { cozc y ctzec (quetz) levantar } \\ \text { cyeu- } & \text { cye'uc (ciauh) cansar } \\ \text { cu- } & \text { cuc (couh) comprar } \\ \text { pu- } & \text { puc (pouh) limpiar } \\ \text { chu- } & \text { chuc (chiuh) hacer } \\ \text { otz- } & \text { ozc (uetz) caer } \\ \text { coch- } & \text { coxc (coch) dormir } \\ \text { quec- } & \text { quec (cac) oir } \\ \text { patan- } & \text { patanc (patlan) volar } \\ \text { molun } & \text { molu'nc (molon) hervir } \\ \text { xaman- } & x a m a^{\prime} n c \text { (xaman) quebrar } \\ \text { caxan- } & \text { caxa'nc (caxan) sanar } \\ \text { temi- } & \text { temc (tlan) acabar } \\ \text { moyan- } & \text { moya'c (yan) esconderse } \\ \text { apec- } & \text { apec entrar }\end{array}$

Mexicano

Presente pitza $m a$ mama toma quiça quetza ciaui coa poui chiua uetzi cochi cachi patlani moloni xamani caxani tlami yana

En esa clase se encuentran los verbos en $o a$ del mexicano clásico cuyas raices terminan en $\grave{o}$, y los en $i a$ cuyas raices terminan en $\grave{i}$.

pelupelu'c (palo) lamer pib'lu- paloa pipiloa

\begin{tabular}{|c|c|c|}
\hline \multicolumn{2}{|c|}{ Pochutla y Mexicano } & MEXICANO \\
\hline Raiz & Pretérito & Presente \\
\hline polu- & polu'c (polo) perder & poloa \\
\hline tepu- & tepo'c (tlapo) abrir & tlapoa \\
\hline tzulu- & tzulu'c (tzolo) sacudir & tzoloa \\
\hline hulu- & $h u l u^{\prime} c$ pepenar & \\
\hline$i x q u e^{\prime}$ & $i x q u e^{\prime} c$ (ixqui) asar & $i x q u i a$ \\
\hline mexti & mexti'c (maxti) enseñar & maxtia \\
\hline mocti & mocti'c (micti) matar & mictia \\
\hline mamui' & mamui'c bañar & \\
\hline tati' & 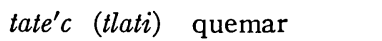 & tlatia \\
\hline tali' & 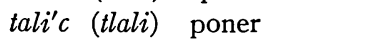 & tlalia \\
\hline temi' & temi'c (tlami) acabar & tlamia \\
\hline namocti' & namocti'c (namicti) casarse & namictia \\
\hline tzupini' & tzupini'c (tzupini) picar & tzupinia \\
\hline$q u i x i^{\prime}$ & quixi'c sacar & \\
\hline$i l p i^{\prime}$ & ilpi'c (ilpi) atar & ilpia \\
\hline$i t i^{\prime}$ & iti'c (ito) decir & itoa \\
\hline
\end{tabular}

Los verbos cuyas raices terminan en $d$ son también de esa clase:

qua quac $(q u a)$ comer qua

Los verbos cuyas raices terminan en $c$ y que toman $a c$ en mexicano, no toman sufijo en Pochutla.

$\begin{array}{cll}\text { pec- } & \text { pec (pac y pacac) lavar } & \text { paca } \\ \text { mec- } & \text { mec (macac) dar } & \text { maca } \\ \text { talvez } & & \\ \text { apec- } & a p e^{\prime} c \text { entrar } & \end{array}$

Los verbos del mexicano clásico que añaden $c$ a la $a$ del presente, o a la $o$ (sin saltillo) de la raiz, añaden en Pochutla $c$ a la $e$ del imperativo.

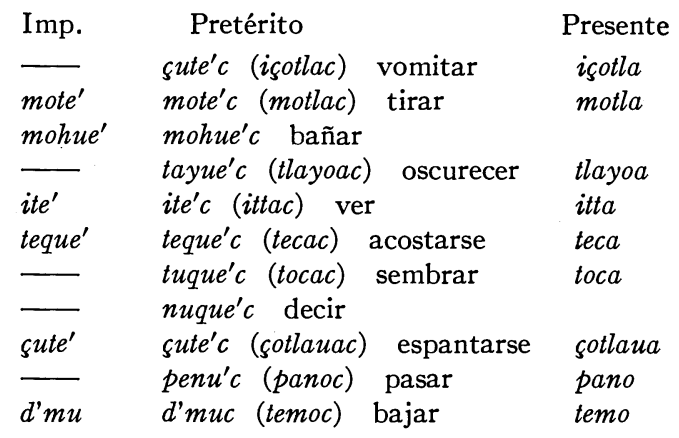

Verbos que en el mexicano clásico terminan en $i$ y toman la $c$, en Pochutla cambian la $i$ que llega a ser $o$. 


\begin{tabular}{|c|c|}
\hline \multicolumn{2}{|r|}{ Pochutla y Mexicano } \\
\hline Presente & Pretérito \\
\hline aci & $a_{c ̧ o^{\prime} c}$ (acic) encontrar \\
\hline & $\begin{array}{l}\text { tatzo'c (tzatzic?) gritar } \\
c u g^{\prime} l u^{\prime} c \text { hace frio }\end{array}$ \\
\hline
\end{tabular}

\section{FORMACIÓN DEL PRESENTE}

Los verbos que terminan en $a$ en el mexicano clásico, tienen la misma terminación en Pochutla.

\begin{tabular}{|c|c|c|}
\hline Raiz & Presente & \\
\hline tom- (tom-) & toma' $\quad$ toma $)$ & desatar \\
\hline nutz- (notz-) & $n u t z a^{\prime} \quad(n o t z a)$ & llamar \\
\hline$m e c-(m a c-)$ & $m e c a^{\prime} \quad($ maca $)$ & dar \\
\hline tayu- (tlayo-) & tayua' (tlayoa) & obscurecer \\
\hline$i t-(i t t-)$ & $i t a^{\prime} \quad(i t t a)$ & ver \\
\hline$n u c-$ & $n u c a^{\prime}$ & decir \\
\hline chu- (chiuh-) & chua' (chiua) & hacer \\
\hline chuc- (choc-) & chuca' (choca) & llorar \\
\hline quiz- (quiz-) & quiça' (quiça) & salir \\
\hline cuizc- & cuizca & tirar \\
\hline cotz- (quetz-) & ctza (quetza) & levantar \\
\hline temo- (temò-) & temoa' (temoa) & buscar \\
\hline teto- (tlàtò-) & tetoa' (tlàtoa) & bramar \\
\hline tag'lu- (cuilò-) & tag'lua' (cuiloa) & escribir \\
\hline cu- (couh-) & $c u a^{\prime} \quad(c o a)$ & comprar \\
\hline
\end{tabular}

Raices que terminan en $a ̀$ no toman $a$ en el presente.

\begin{tabular}{lll}
\multicolumn{2}{c}{ Raiz } & \multicolumn{2}{c}{ Presente } & \\
ma- (mà-) & ma (ma) & tomar \\
mama- (mamà-) & mama (mama) & cargar \\
$q u a-(q u \grave{a}-)$ & $q u a(q u a)$ & comer
\end{tabular}

Las raices en $i$ que toman $a$ mexicano, no tienen sufijo en Pochutla.

\begin{tabular}{lll}
\multicolumn{2}{c}{ Raiz } & \multicolumn{1}{c}{ Presente } \\
machti- & mextí (machtia) & enseñar \\
namicti- & namocti (namictia) & casarse \\
tzopini- & tzupini (tzopinia) & picar \\
tzilini- & tzilini' (tzilinia) & sonar
\end{tabular}

Otros verbos cuyas raices terminan en $i$ y $o$ sin saltillo no tienen sufijos, ni en el mexicano clásico ni en Pochutla.

$\begin{array}{lc}\text { Pochutla } & \text { Mexicano } \\ \text { aci' } & \text { aci encontrar } \\ \text { tatzi } & \text { tzatzi (?) gritar }\end{array}$

PoChutLA
yequi'
nqui
tqui
calamqui'
d'mu

Mexicano

- llover

nequi querer

(tequiti) llevar

- acordarse

temo bajàr

Los verbos cuyas raices terminan con una consonante y que toman $i$ en el mexicano clásico, toman el mismo sufijo en Pochutla.
Raiz

caxan- (caxan-)

quec- (cac-)
Los en $e$ en el dialecto de Pochutla no toman sufijos aunque en el mexicano clásico tomen $a$.

PochutLa
che
çute' $^{\prime}$

Mexicano
chia
çatlaua

Presente

$\begin{array}{lll}\text { caxani' } & \text { (caxani) } & \text { sanar } \\ \text { quequi' } & \text { (caqui) } & \text { oir }\end{array}$

FORMACIÓN DEL IMPERATIVO Y DEL FUTURO

El imperativo de todos los verbos cuyo presente toma el sufijo $a$, toma $e$; y el futuro se forma anadiendole $z$ al imperativo.

\begin{tabular}{|c|c|c|c|}
\hline \multicolumn{2}{|c|}{ Pochutla } & \multirow{2}{*}{$\begin{array}{c}\text { Mexicano } \\
\text { Presente }\end{array}$} & \\
\hline Imperativo & Futuro & & \\
\hline & apitze'z & apitza & purgar \\
\hline moyane' & moyane'z & yana & esconderse \\
\hline peque' & peque'z & paca & lavar \\
\hline pitze' & $\longrightarrow$ & pitza & soplar \\
\hline & poxque'z & pixca & pizcar \\
\hline metze' & metze'z & - & sentarse \\
\hline meque' & $\longrightarrow$ & maca & dar \\
\hline mote & mote'z & motla & tirar \\
\hline mohue' & mohue'z & $\longrightarrow$ & bañar \\
\hline tachapane' & $\longrightarrow$ & tlachpana & barrer \\
\hline$i t e^{\prime}$ & $i t e^{\prime} z$ & $i t t a$ & ver \\
\hline teque & teque'z & teca & acostarse \\
\hline tome' & tome'z & toma & desatar \\
\hline & $t u q u e^{\prime} z$ & toca & sembrar \\
\hline & nuque'z & $\longrightarrow$ & decir \\
\hline & $x i x e^{\prime} z$ & xixa & mear \\
\hline & tzaue'z & tzaua & hilar \\
\hline & tzome $e^{\prime} z$ & tzoma & coser \\
\hline che & chez & chia & esperar \\
\hline chue' & & chiua & hacer \\
\hline quane' & $\longrightarrow$ & & rascar \\
\hline queue' & $q u e u e^{\prime} z$ & $\longrightarrow$ & guardar \\
\hline quice' & quice'z & quiça & salir \\
\hline cuique' & $\longrightarrow$ & cuica & cantar \\
\hline ctze & ctzez & quetza & levantar \\
\hline
\end{tabular}


Verbos cuyas raices terminan en $o, \grave{o}, i$ en el mexicano clásico no toman sufijo en el imperativo, y añaden $z$ a la raiz en el futuro.

\begin{tabular}{|c|c|c|c|}
\hline $\begin{array}{r}\text { Росне } \\
\text { Imperativo }\end{array}$ & $\begin{array}{l}\text { LA } \\
\text { Futuro }\end{array}$ & $\begin{array}{l}\text { Mexicano } \\
\text { Presente }\end{array}$ & \\
\hline imperacivo & paxalu'z & rresemte & pasearse \\
\hline pech $u^{\prime}$ & - & pechoa & apretar \\
\hline pelu' & & paloa & lamer \\
\hline pib'lu' & & pipiloa & envolver \\
\hline tepu $u^{\prime}$ & & tlapoa & abrir \\
\hline & telu'z & tlaloa & correr \\
\hline tzulu' & & tzoloa & sacudir \\
\hline hulu' & & & pepenar \\
\hline tecu' & $t e c u^{\prime} z$ & tlèco & subir \\
\hline$d^{\prime} m u$ & $d^{\prime} m u z$ & temo & bajar \\
\hline petebi' & {[} & & ayudar \\
\hline pig'li' & pig'le'z & $\longrightarrow$ & golpear \\
\hline & mexti'z & machtia & enseñar \\
\hline mocti ${ }^{\prime}$ & & mictia & matar \\
\hline mamui' & 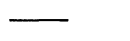 & - & bañar \\
\hline tatenli' & tatenli'z & tlàtlania & preguntar \\
\hline tati & 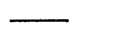 & tlatia & quemar \\
\hline tali' & tali'z & tlalia & poner \\
\hline temi' & & tlamia & acabar \\
\hline totoqui' & & toquia & atizar \\
\hline namig'li' & - & (namaquiltia) & vender \\
\hline- & namocti'z & namictia & casarse \\
\hline$q u i x i^{\prime}$ & $q u i x i^{\prime} z$ & quixia & sacar \\
\hline$i l p i^{\prime}$ & $i l p i^{\prime} z$ & ilpia & atar \\
\hline & xamani'z & xamani & quebrar \\
\hline
\end{tabular}

Los verbos cuyas raices terminan con una consonante y toman $i$ en el presente, tienen el sufijo $z$ en el futuro. No pude apuntar imperativos de ese grupo.

$\begin{array}{ll}\text { Pochutla } & \text { Mexicano } \\ \text { Futuro } & \text { Presente } \\ \text { pata'nz } & \text { patlani } i \quad \text { volar }\end{array}$

Los verbos cuyas raices terminan en $i$ sin saltillo la cambian en $o$ en el imperativo y futuro (véase el mismo cambio en el pretérito, p. 20).

\section{Pochutla}

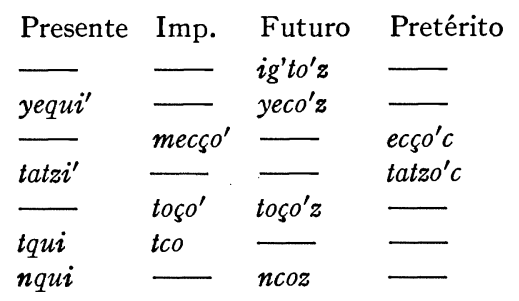

Pochutla

Mexicano

Presente Imp. Futuro Pretérito Presente

calamqui' - calamco'z- - acordarse

$-\quad-\operatorname{cocho}^{\prime} z-\quad$ cochi dormir

cug'li' - cug'lu'c - hacer frio

$($ (apequi') apeco' apeco'z ape't - entrar

\section{FORMACIÓN DEL IMPERFECTO}

El imperfecto se forma añadiendo el sufijo $y a$ al imperativo.

$\begin{array}{ll}\text { nuca' } & \text { nuqueya decía } \\ a i^{\prime} & \text { ntzaçoya te encontraba } \\ (\text { otzi }) & \text { otzoya caía } \\ n q u i & \text { nconcoya lo quería } \\ i t i^{\prime} & \text { nichitiya me decía } \\ \text { bia' }^{\prime} & \text { ncobeya lo tenía }\end{array}$

FORMAS COMPUESTAS CON LOS VERBOS DE IR, VENIR Y ESTAR

Encontré dos formas del "Gerundio" con "ir," el imperativo y el pretérito. El imperativo tiene el sufijo $t i$ (mexicano $t i$ ).
ximoti' at itrae agua!
xtag'luti' ivete a escribir!
xtemuti' ianda, buscalo!
xtaquati' ivete a comer!
xicueti' ivete a comprar!
xucochoti' ianda, duerme!

El pretérito tiene el sufijo $t u$ (mexicano to).

$\begin{array}{ll}\text { tyac paxalutu' } & \text { fuiste a pescar } \\ \text { yac tapequetu' } & \text { fué a lavarlo } \\ \text { yac mamuitu' } & \text { fué a bañar }\end{array}$

Tengo solamente el imperativo del gerundio con "venir," que tiene el sufijo qui (mexicano qui).

$$
\text { xtaquaqui' ivente a comer! }
$$

En el perfecto siempre se usa el gerundio con "ir" en vez del con "venir."

enola'c ntzimequetu' vine a darte

enola'c tixtacuetu' vine para que me pagues

Las formas en tuc (mexicano $t i+o c$ ) se usan mucho.
ntapotu'c estoy contando
tatzotu'c está gritando
tag'lutu'c está escribiendo 
moztemutu'c están buscándote

ntatzontu'c estoy cosiendo

cocoxtu'c está durmiendo

enquig'totu'c estoy tejéndolo

titzintu'c está naciendo

$n t z o c h e t u^{\prime} c$ estoy esperándote

quiztu'c está saliendo

ntacuiquetu'c está cantando

tacoztu'c está platicando

moluntu'c está hirviendo

Todas las formas añaden el sufijo al imperativo.

PREFIJO DEL PRETÉRITO

El pretérito toma el prefijo $e$ (mexicano $o$ ) el cual prefijo no se junta firmemente con el verbo.

enopib'lu'c ya me cubrí
etapig'le c golpeó
encupu'c lo limpié
enola'c vine
enctamote'c ya lo tiré
ecmoctiqui't ya lo mataron

\section{REDUPLICACIÓN}

Ya se mencionaron los plurales de sustantivos que reduplican la primera sílaba. Ejemplos de verbos frecuentativos con reduplicación son:

$$
\begin{aligned}
& \text { pib'loa envolverse } \\
& \text { totoqui' atizar } \\
& \text { tutuca' correr } \\
& \text { cocoxtu'c está durmiendo }
\end{aligned}
$$

También debemos mencionar

$$
\text { yulicyuli'c despacio }
$$

\section{VERBOS IRREGULARES}

Encontré las formas siguientes del verbo irregular $u i$ irse.

PochutLA
$n u i^{\prime}$
$t u i^{\prime}$
$u i^{\prime}$
$t u i^{\prime}$
$u i^{\prime}$
unya'c
tia'c, $t u i^{\prime} c$
uya'c

Mexicano
niauh me voy
tiauh te vas
yauh se va
tiaui, tiui nos vamos
yauih, iuh se van
onia me fuí
otia te fuiste
oya se fué

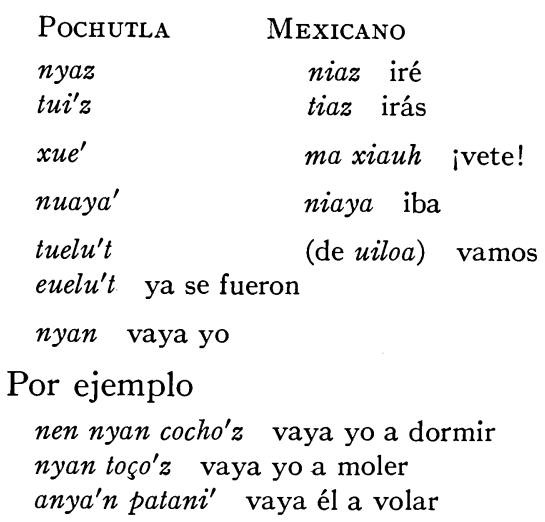

Encontré pocas formas del verbo $u i^{\prime} t z$, venir.

$\begin{array}{lc}n u i^{\prime} t z & \text { ninuitz vengo } \\ \text { tui'tz } & \text { tiuitz vienes } \\ u i^{\prime} t z & \text { uitz viene } \\ & \text { VERBO GUALAC, VENIR } \\ \text { nola'c } & \text { oniuala vine } \\ \text { tola'c }_{\text {guala'c }} & \text { otiula veniste } \\ \text { tolaqui't } & \text { ouala vino } \\ \text { leca' } & \text { otiualaque venimos } \\ & \text { (xinalacan ?) ivente! }\end{array}$

\section{COMPOSICIÓN}

Encontré un número pequeño de vocablos que demuestran que los métodos de composición del dialecto de Pochutla y del de México eran iguales.

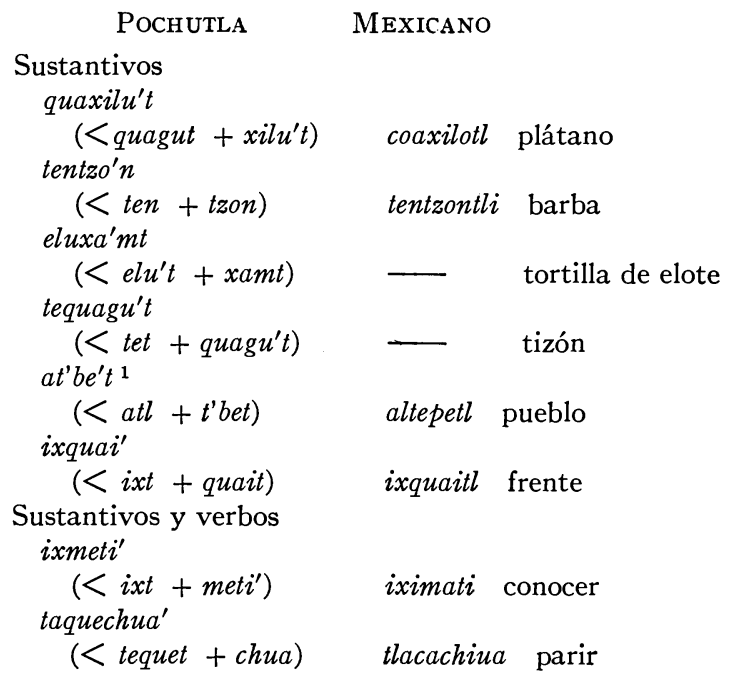

1 No oigo yo la $l$, aunque en el vocabulario de Apolonio Rosario se encuentra. 


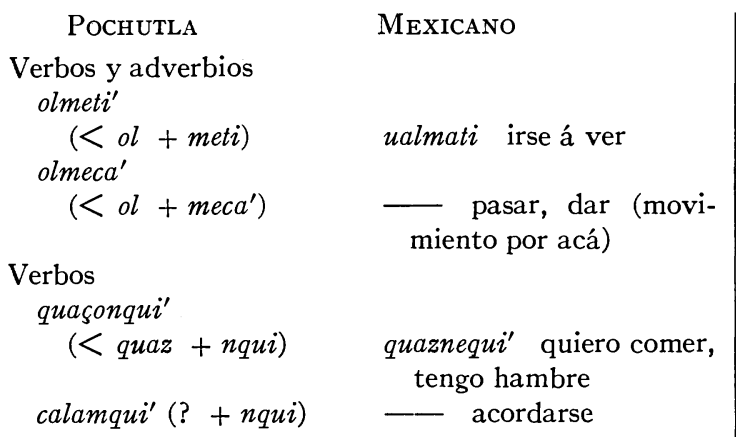

\section{VOCABULARIO}

ABREVIATURAS
A Andrea Castillo

El Eleuterio Avesilla

Ep Epifanio Pina

Es Estanislao Pina

F Feliciana

Fr Francisca

I Ines Vázquez

Jo Joaquina

pr. presente

p. pretérito

f. futuro
M Mauricia Riquel

Mr Maria Trinidad

P Paula Nicha

Pe Pedro Marcelino Pastor

S Sabina Martínez

imp: imperativo impf. imperfecto ger. gerundio

\section{POCHUTLA-CASTELLANO}

\section{ayago' (mex. ayac) no hay F M P S}

az nui' Uetu'l ampa ayago' tumi'n no me

voy a Huatulco porque no hay dinero F M quineba' ayagoai' no está aquí S ayago' nintega' no hay nada F M ite ayago' moye'? ¿no está tu madre? S $a y u^{\prime} t$ (mex. $\left.\bar{a} y o t l\right)$ tortuga F M ay $t e^{\prime}$ (mex. aya tle) ¡no! A I Es Mr P S (hayte Apolonio Rosario) ay $t e^{\prime}$, tiome' $^{\prime}$ no, después I ay $t e^{\prime}, a z n u i^{\prime}$ no, no me voy A ay $t e^{\prime}, a z$ nococoa' no, no estoy enfermo

$\mathrm{MrS}$

ay $t e^{\prime}$, COMPADRE $\mathrm{J}$ ¡buenos días, compadre! (?)

$a u e^{\prime} c$ (véase mex. uacqui) mojarse $\mathrm{M} \mathrm{MrS}$.

Véase uac. Según esa forma la $a$ de $u \bar{a} c q u i$ SECo es larga, la de uaqui MOJARSE breve $a o^{\prime} c$ aue'c mucho se mojó S naue'c me mojé S

aueque't (mex. ahuacatl) aguacate $\mathrm{S}$ $a p a^{\prime} z t$ (mex. āpaztli) olla A F Fr Jo M Mr P S noapa'z mi olla Fr Jo

exama'nc napa'zt se quebró la olla $\mathrm{S}$ apeco', imp.; eyape'c, ape'c; apeco'z entrar A F M Mr P S

$a p e^{\prime} c$ ce $u i^{\prime} z t i$ noxo' $i$ me entró una espina en el pié.

eyape c ito'c quagu't ya entró en la cárcel $\mathrm{P}$ eyape'c ogüe'l ya entró la noche $\mathrm{Mr} \mathrm{S}$ eyape'c tune'l ya se ha puesto el sol $\mathrm{P}$ $n e b a p e^{\prime} c$ (<neba' $\left.a p e^{\prime} c\right)$ aquí entró $\mathrm{S}$ xapeco' ¡entra! A F M S $n u i^{\prime}$ napeco'z voy a entrar S apitze'z f. (mex. apitza) purgar nui' napitze'z voy a purgar apoto'ct (mex. ipotoctli) humo Mr S $a m e^{\prime} t$ (mex. āmatl) papel S; carta S $a^{\prime} m p a$ (mex. $\left.i p a m p a\right)$ porque A F M Mr P S xmuyane' ampa moztemutu'c escóndete porque te están buscando $\mathrm{S}$

az nyac ampaço'c (< ampa aço'c) nichota' nogüelu' no me fuí porque mucho me miró mi marido $\mathrm{P}$

naco' igüe'n uya'c. ¿̇teca'? ampaço'c chuca' $i b^{\prime} l u^{\prime}$ hace poco tiempo se fué.¿Por qué?-Porque llora su hijo $\mathrm{P}$

nonocoa' noliu' a'mpa emo'c nob'lu' me duele el corazón porque se murió mi hijo $\mathrm{F} \mathrm{M}$ $m u e^{\prime} n$ tui $a^{\prime} m p a$ tibia' tumi'n tu te vas porque tienes dinero $\mathrm{F} \mathrm{M}$

nen unya'c a'mpa naguaçonqui' me fuí porque tengo hambre $\mathrm{M} \mathrm{MrS}$

¿teca'? a'mpa az ncobia' tumi'n ¿por qué?Porque no tengo dinero $\mathrm{P}$

at (mex. $\bar{a} t l$ ) agua F Jo M Mr S (ad Apolonio Rosario)

ate'n (mex. atentli) río $\mathrm{F}$ Jo $\mathrm{M} \mathrm{P} \mathrm{S}$ (adem Apolonio Rosario)

$a n t u^{\prime}$ ate' $n$ ¡vámonos al río! S

tutuca' ca $x u e^{\prime}$ ate' $n$; que pronto vayas al río! $\mathrm{F} \mathrm{M}$ 
$a t^{\prime} b e^{\prime} t$ (mex. altepetl) pueblo $\mathrm{Mr} \mathrm{S}$ (altibet

Apolonio Rosario)

noat'be'u mi pueblo $\mathrm{S}$

mat'be'u tu pueblo $\mathrm{S}$

(alvuna mar, Apolonio Rosario)

ato'mt (mex. atemitl) piojo S

ato'lc fuera F M P S

mato'lc $(<m a$ ato'lc) allí fuera P S

tiquiça' mato'lc ¿sales fuera? S

xiquice' mato'lc isale fuera! S

ma quet ato'lc está fuera F M

$a n y e^{\prime} n$ (?) (véase $u i^{\prime}$ irse)

$a n y e^{\prime} n n u i^{\prime} U_{a x e} c$ no he ido a Oaxaca S anye' $n$ totoqua'c ¿no has comido? $\mathrm{S}$

$a n t u^{\prime}$ ¡vámonos! A Es F Fr I Jo M Mr P S antu' totaqua'z ¡vámonos a comer! A $\mathrm{P}$ $a n t u^{\prime}$ leca' ¡vámonos pronto! F M (véase leca' venir)

antu' $u^{\prime}$ a toch $a^{\prime} n$ ¡vámonos a nuestra casa!

Jo

az no A F Fr I Jo M Mr P S

az nui' no me voy I M Mr S

az ncobia' teyu'l no tengo maiz $\mathrm{P}$

az tinqui' no quieres $\mathrm{P} \mathrm{S}$

$a z$ cota $^{\prime}$ no lo vé $F \mathrm{M}$

te az tiba' ce tila'n ¿no tienes un pollo? $\mathrm{S}$ ni az nui'z naco', quago' nui'tz si no vengo

ahora, vengo mañana $\mathrm{S}$

xite' na conebo'l que az chuque' ¡mira al

niño que no llore! F M

ay $t e^{\prime}$, az nococoa' no, no estoy enfermo

$\mathrm{MrS}$

$a c i^{\prime}$; $a c^{\prime} o^{\prime}$; $a c ̧ o y a^{\prime}$ (mex. aci) encontrar

SI $a z t_{u i^{\prime}}$ naco nime $^{\prime} n$ az caci' si no te vas

orita, no lo encuentres ( $c a c i^{\prime}$, sin sujeto

de la segunda persona) $\mathrm{Mr}$

ma ntzaci' allí te encuentro $\mathrm{S}$

$n e b a^{\prime}$ tichaci' aquí me encuentras $\mathrm{S}$

encaşo'c ya lo encontré F M S

tiço'n nonaço'c neba', ntzochetu'c llegué

aquí, estoy esperándote $\mathrm{S}$

ntzaçoya' te encontraba $\mathrm{S}$

$a$ şo $^{\prime} c$ (mex. $a c ̧ o$ ) mucho, muy A F Fr I Jo

M Mr P S (asot, asoc Apolonio Rosario) aço'c tamoca' noliu' mucho me duele el corazón F $\mathrm{P}$ $a c ̧ o^{\prime} c$ tacho'm unyo'c neba' aquí hay muchos perros $\mathrm{S}$

$a c^{\prime} o^{\prime} c$ unti' muy borracho $\mathrm{S}$

$a c ̧ o^{\prime} c$ unyo'c hay muchos $\mathrm{MrS}$

$a o^{\prime} c$ unyo'c cue't hay muchas culebras

F M

achiquelo'm $\mathrm{P}$ S, azquelo'm $\mathrm{F}$ M camarón

(axt) noachu' (mex. ächtli) mi semilla Fr Jo ah na (mex. auh ini) entonces

te tichemeca', ah na nui' dame cosa, entonces me voy $\mathrm{M} \mathrm{S}$

$a c$ (mex. $\bar{a} c$ ) ¿quién? F M P S

ac totactza' ¿con quién hablas? F M

ac nacona' ¿quién es ese? $\mathrm{P}$ (ac nacona

Apolonio Rosario)

ac nuca' ¿quién dijo eso? $\mathrm{F} \mathrm{M}$

ac mozmexti' LA IDIOMA ¿quién te enseñó

el idioma? S

ac mozpetebi'c ¿quién te ayudó? $\mathrm{S}$

$n u e^{\prime} n$ tite'z ac timeca' verás a quién le des $\mathrm{S}$ (agueneumi, andar, Apolonio Rosario)

aque't (mex. $\bar{a} c a t l$ ) carrizo S

algua' (mex. yalhua) ayer M Mr S (algua

Apolonio Rosario)

algua' tolaqui't mañana vendremos $\mathrm{S}$

$e y o^{\prime} m$ (mex. yei) tres Ep M Mr S oyo'm A

(eyom Apolonio Rosario)

eyu't (mex. ayòtli) calabaza F Fr Jo M

(eyud Apolonio Rosario)

eueta'c (mex. huehue) está viejo F M (eguetac

Apolonio Rosario)

$a c ̧ o^{\prime} c$ toeueta'c estás muy viejo $\mathrm{P}$

eti' (mex. etic) pesado M Mr S

$a z$ eti', nen cotqui' no está pesado, puedo

llevarlo $\mathrm{S}$

etiote'c P, teote'c P (mex. teotlac) tarde

(eyudeu Apolonio Rosario)

nui' a'mpa aço'c etiote' me voy porque es muy tarde $\mathrm{P}$

$a$ ço $^{\prime} c$ etiote $^{\prime} c$ ya es muy tarde $\mathrm{P}$

etu'l (mex. atolli) atole F I M Mr S

motolu' tu atole $\mathrm{Mr} \mathrm{S}$

eat (mex. eztli) sangre F M (est Apolonio

Rosario)

(escocul, arco, Apolonio Rosario) 
eç̧o'c (mex. icucic) cocido, maduro $\mathrm{M}$ Mr S meç̧o' ¡cuécelo! S

elu't (mex. elotl) elote Mr S

iye'c (mex. iyac) apesta $\mathrm{A}$

$i y e^{\prime} c$ tacho' $m$ apesta el perro A

$i t a^{\prime}$ pr.; ite' imp.; ite'c p.; ite'z f. (mex. itta) ver A F I M MrP S

xte ce cue't ¡mira, una culebra! M Mr S xite ¡mira! A

xite' na conebo'l ¡mira, el niño! F M

nichota' él me mira $P$

tichota' tú me miras F M

ecote'c ya lo vió F M

az tite $^{\prime} c$ ¿no lo viste? S

az tichote'c ¿no me viste? S

az nichote'c ¿no me vió? $\mathrm{P}$

encote'c $^{\prime}$ ya lo ví $\mathrm{P}$

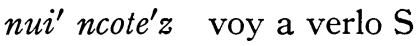

$m u e^{\prime} n$ tite' $z$ vas a verlo $\mathrm{S}$

tite'z ya verás I

nen ncote'z nomi'l voy a ver mi milpa $\mathrm{P}$

$n u i^{\prime} n$ cote $^{\prime} z$ nocOMPALE'$u$ voy a ver $\mathrm{mi}$ compadre I

ite'nc (mex. itlan) debajo S

ite'nc apa'zt debajo de la olla S

iti, eti; iti'c p.; itiya' impf. (mex. itoa) decir

$\mathrm{M}$ Mr P S

igüe'n nicheti' ella me dijo $\mathrm{P}$

te motzeti'c ¿qué te dijo? M Mr S

te ticheti'c ¿qué me dijiste? $\mathrm{S}$

az ntzeti'c az monamocti' ¿no te dije que no te cases? S

$u e^{\prime}$ na ntzeti'c eso es lo que le dije S

nicheti'c noye' me dijo mi madre $\mathrm{S}$

nichitiya' noye' na IDIOMA me hablaba mi madre el idioma $\mathrm{S}$

igüe'n (mex. yehuatl) él F M P (iguin Apolonio Rosario)

az conqui'gü̈'n (<conqui' igüe' $n$ ) él no quiere $\mathrm{F}$

igüe' $n$ nicheti' ella me dijo $\mathrm{P}$

(iuit ?) noibe' (mex. icuitl ?) mi hermana.

Talvez se debe escribir noiue ${ }^{\prime}$ en vez de noibe'

ina' (mex. inin) éste A S (inac Apolonio Rosario) quet MAS BUENO $i n a^{\prime}$ o $n a m i^{\prime} n$ ¿está mejor éste o ése? S

ina' conebo'l quixi'c itecu' este muchacho es parecido a su padre $\mathrm{S}$

ina' neque't quet MANIDU esta carne está manida $\mathrm{S}$

inane'l teque't aço'c picho't este hombre está muy viejo A

entzute'c p. (mex. içotla) vomitar S

entzute'c na conebo'l vomitó el niño $\mathrm{S}$

(ixt) moi'x (mex. ixtli) tu cara Mr S (mix

Apolonio Rosario)

(mixcuay $[<i x t+q u a i t]$ tu frente, Apolonio Rosario)

$i x i^{\prime} c$ semejante, parecido $\mathrm{Mr} \mathrm{S}$

ina' conebo'l quixi'c itecu' este muchacho

es parecido a su padre $\mathrm{S}$

$a \varsigma^{\prime}{ }^{\prime} c i x i^{\prime} c$ itecu' muy parecido a su padre $\mathrm{Mr}$

(ixtotolu't) (mex. ixtololòtli) ojo F M $\mathrm{Mr}$

$\mathrm{Pa} \mathrm{P} \mathrm{S}$ (extodolu Apolonio Rosario)

noxtotolu' mi ojo F M Mr P Pa

nixtotolu' ${ }^{\prime}$ mi ojo S

ixtotolu' su ojo F M

$i x n a^{\prime} c$ (mex. ixnauac) frente. Véase nac

$x$ moteque $i x n a^{\prime} c$ quagu't jacuéstate frente

al banco! $\mathrm{S}$

ixque' imp.; ixque'c p. (mex. ixquia) asar $\mathrm{M}$

$\mathrm{MrS}$

xixque iásalo! M Mr S

xixque $e^{\prime}$ a quaxilu't PA tiquazqui't asa el

plátano para que lo comamos $\mathrm{S}$

enoxque'c ya está asado $\mathrm{M} \mathrm{Mr} \mathrm{S}$

ic (mex. ic) ¿cuándo? A F M Mr S

ic tui'z ¿cuándo te vas? F M

ic tinamocte' ¿cuándo te casas? Mr S

ic tola'c ¿cuándo vinieron? $\mathrm{S}$

icualgua', igualgua'(?) antier M MrS. Véase algua

(icoz-) (mex. quiquiçoa ?) chiflar S

nen nicoztu'c estoy chiflando

$i c p a^{\prime} c$ (mex. icpac) sobre S

$i c p a^{\prime} c$ nomi'l en mi milpa

tacpa'c sobre

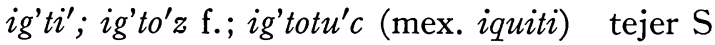

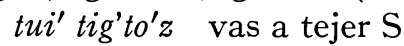


$n u i^{\prime}$ nig'to'z ce tzoct voy a tejer un paño $\mathrm{S}$ enquig'totu'c estoy tejiéndolo $\mathrm{S}$

ilpi' imp.; ilpi'c p.; ilpi'zf. (mex. ilpia) atar

$\mathrm{M} \mathrm{MrS}$

xilpi ¡átalo! M Mr S

encolpi'c lo até $\mathrm{S}$

$m u e^{\prime} n$ tilpi'c tú lo ataste $\mathrm{S}$

$n u i^{\prime}$ ncolpi'z voy a atarlo S

(ya ?); moyane' imp.; enoya'c p.; moyane'z f.

(mex. inaya, yana ?) esconderse $\mathrm{S}$

xmoyane' iescóndete! S

xmoyane' a'mpa moztemutu'c escóndete,

porque están buscándote $\mathrm{S}$

enoya'c estoy escondido $\mathrm{S}$

noyane'z voy a esconderme S

(yajai, alas, Apolonio Rosario)

(yactangui, olvidar, Apolonio Rosario)

(ye) madre F Fr M Mr S

noye' mi madre F Fr M Mr S

moye' tu madre F Fr M Mr

iye' su madre F M

(yeque't [mex. yacatl] nariz F M Mr S)

noye'c mi nariz S (mec, tu nariz, Apolonio

Rosario) F y M dijeron $n o n e^{\prime} c$ MI NARIz, lo que es une equivocación; otra vez dijeron mec, tu nariz, como Apolonio Rosario; evidentemente eso también era une equivocación

yect, yequi pr.; yectu'c p.; yeco'z f. lluvia.

(Véase mex. ectal, viento?) M Mr S

(yexixiltud, yeexniduc Apolonio Rosario) me $u i^{\prime} t z$ yect allí viene lluvia $\mathrm{Mr} \mathrm{S}$

nichoma'c yect me cogió la lluvia $\mathrm{M} \mathrm{Mr}$

yectu'c está lloviendo Mr S

$u i^{\prime}$ yeco'z va a llover S

yequi' llueve $\mathrm{S}$

yut, viento, (yud Apolonio Rosario) S

yut tetoa' brama el viento $\mathrm{S}$

yulicyuli'c (mex. yolic) despacio M Mr S

yulicyuli'c ui' caxa'nz sana poco a poco

$\mathrm{M} \mathrm{MrS}$

yulicyuli'c xapeque' na apa'zt ¡lava la olla!S

ogüel (mex. yoalli) noche Mr S

eyape'c ogüe'l ya entró la noche Mr S

pen ogüe'l anoche S opque't (mex. icpatl, metátesis de la $c p$ ) hilo Fr Jo M Mr P S

xitzuqua' na opque't ¡corta el hilo! S

nopcu' mi hilo Fr Jo S

ome'm (mex. ome), dos A Ep M Mr P S (omem

Apolonio Rosario)

xima' ome'm tito't ¡toma dos huevos! $\mathrm{Mr}$ $o m e^{\prime} m$ cobia' JOAQUINA Joaquina tiene dos $\mathrm{S}$ omeme't quig'lazqui't dos mujeres $\mathrm{P}$

ot (mex. omitl), hueso $\mathrm{M} \mathrm{Mr} \mathrm{S} \mathrm{(tood,} \mathrm{nuestro}$ hueso, Apolonio Rosario)

$o t^{\prime} c a^{\prime} n$ (mex. òtli) camino Mr S. Véase mex. ottica en el camino

otca' $i$ dulce $\mathrm{S}$

ozte't (mex. iztatl) sal F I M Mr Pa (oste't Apolonio Rosario)

(oxt) (mex. iztetl)

moxt tu uña $F$ M. Se pronunció clara-

mente moxt, no mozt. Apolonio Rosario

también escribe moxt

$o x c a^{\prime} z t$ jícara F Fr M Mr S

oxque't (mex. ixcatl) algodón F Fr Jo M Mr

$\mathrm{P}$ S

noxcu' mi algodón $\mathrm{F} \mathrm{M}$

moxcu' tu algodón $\mathrm{S}$

(otz-);ozc p.; otzo'z f. (mex. uetzi) caer S

ozc cayó $\mathrm{S}$

nozc caí $\mathrm{S}$

$n u i^{\prime}$ noctzo'z (sic) voy a caer S

nui' noctzoya' (sic) iba a caer S

oco't (mex. ocotl) ocote Mr S

oco'xt (mex. oquichtli) hombre $\mathrm{Mr} \mathrm{S}$

quizco'm oco'xt ¿cuántos varones? $\mathrm{Mr}$

$U_{a x e} c$ Oaxaca A F Fr Jo M

$\left(u a^{\prime} c\right)$; euac p. (mex. uaqui) seco S. Véase

auec

$e u a^{\prime} c$ xut están secas las hojas

Uetu'l Huatulco F M Mr S

$u e^{\prime} l$ (mex. uel) poder $\mathrm{S}$

az uel noctza'n no se puede levantar S

$u i^{\prime}$ (mex. yuah) ir. Véase p. 23 A El F Fr

I M Mr P S

nui' ncutuque'z teyu'l voy a sembrar maiz I

nen az nui' no me voy F M MrS

ic tui' ¿cuándo te vas? $\mathrm{MrS}$

$u l i^{\prime} c t u i^{\prime}$ ique vayas bien! F M 
ca tui' quago' ¿a dónde te vas mañana?

$\mathrm{MrS}$

$m a u i^{\prime} c u e^{\prime} t$ allí va una culebra $\mathrm{S}$

$u i^{\prime} p a t a^{\prime} n z$ va a volar S

nocho' tui todos nosotros vamos F M

az tuilu't no iremos A

nen nconcoya' nyaz quería irme $\mathrm{P}$

ic tui'z ¿cuándo te vas? F M

nen unya'c pen tepo'x me acosté (fuí a mi cama) A

unya'c, PERO nichoma' yect me fuí, pero me cogió la lluvia $\mathrm{M}$

ca $t_{i a} c$ ¿dónde fuiste? (ic tui'c ¿cuándo fuiste? M)

ticon $t i a^{\prime} c$ ¿a qué hora te fuiste? S

$u y a^{\prime} c$ pata'nc se voló S (uyac Apolonio

Rosario)

ca uya'c motecu' ¿a dónde se fué tu padre?

F M

ma nuaya' nozc allí andando caí $\mathrm{S}$

nen nyan cocho'z voy a dormir F $\mathrm{M}$

nyan toço'z voy a moler Jo

nyam patani' voy a volar $\mathrm{S}$

ca $x u e^{\prime}$ ¡anda! vete! A F M Mr S

az tuelu't no nos vamos $\mathrm{P}$

euelu't ya se van $\mathrm{P}$

$u i^{\prime} z t i$ (mex. uiztli) espina F Fr M Mr S

noni'zt mi espina $\mathrm{S}$

$u i^{\prime} t z$ (mex. huitz) venir A F M Mr P S (ehuix

Apolonio Rosario)

ni az nui'z naco', quago' nui'tz si no vengo

ahora, mañana vendré $\mathrm{S}$

naconime'n nui'tz ahorita vengo $\mathrm{P}$

az tui'tz quago' ¿no vienes mañana? $\mathrm{S}$

$m a i^{\prime} t z$ allí viene $\mathrm{F} \mathrm{M}$

ma $u i^{\prime} t z$ totoltzi' $n$ allí viene el cura A S

enui'tz totoltzi'n ya vino el cura $\mathrm{MrS}$

$u i^{\prime} t z$ yect viene la lluvia M S

tuitzelu't venimos A

unyo'c (mex. onoc) estar echado F Fr Jo M

$\mathrm{Mr} \mathrm{S}$

$a$ ço $^{\prime} c$ unyo'c cue't hay muchas culebras

$\mathrm{F} \mathrm{M}$

unti' (mex. iuinti) borracho A F M P S

teca' tounti $m u e^{\prime} n$ ¿por qué te embo-

rrachas? $\mathrm{P}$ $u i^{\prime}$ unti' anda borracho $\mathrm{S}$

untilu't están borrachos $\mathrm{A}$

$u z t i^{\prime}$ (mex. otzti) embarazada P S

$u g^{\prime} l o^{\prime} m$ (mex. ocuilin) gusano A S

$u l i^{\prime} c$ (mex. ueli) bueno Ep F Fr Jo M Mr P

quet uli'c F M uli'c quet Ep está bueno

uluni' imp. (mex. olinia) menear, echar S

xuluni' ca ce quagu't ¡menéalo con un palo! $\mathrm{S}$

az xuluni' napa'zt ique no muevas la olla!

$\mathrm{S}$

xuculuni' ito'c apa'zt jéchalo en la olla! S

$b a$ tener. Véase bia

payo' (castellano paño ?) rebozo, paño Jo

$\mathrm{MrS}$

nopayu' mi rebozo Jo $\mathrm{Mr} \mathrm{S}$

pata'nc p.; pata'nz f. (mex. patlani) volar S

epata'nc voló $\mathrm{S}$

uya'c pata'nc se voló $\mathrm{S}$

$u i^{\prime} p a t a^{\prime} n z$ va a volar S

pate'c (mex. patlauac) ancho S

pan (castellano) pan Fr I Jo Mr

nopanu' mi pan I

(paxalu-); paxalu'zf. (castellano ?) pasearse

$\mathrm{M} \mathrm{Mr} \mathrm{S}$

nui' npaxalu'z voy a pasearme S

yac paxalutu' fué paseándose $\mathrm{S}$

peu'c p. (mex. peua) empezar S

pebe't jicalpezte F M Mr P

petebi' imp.; petebi'c p. ayudar $\mathrm{S}$

ac mozpetebi'c ¿quién te ayudó? $\mathrm{S}$

nixpetebi'c me ayudó $\mathrm{S}$

tixpetebi' jayúdame! $\mathrm{S}$

(pen) (mex. pan) sobre A F Jo M P S

ma quet $i p e^{\prime} n$ MESA está allí en la mesa

F M

$x t e c u^{\prime} i p e^{\prime} n$ na quagu't isube el palo! S

ma quet ipe'n tepoxpo'l está en el tabanco

$n o p e^{\prime} n$ sobre mí S

tope' $n$ sobre nosotros $\mathrm{S}$

$m o p e^{\prime} n$ sobre ti $\mathrm{P}$

$i p e^{\prime} n$ sobre él S

$i p e^{\prime} n$ ogüe'l anoche $\mathrm{S}$

(pen) tiope $n$ (mex. teopantli) iglesia S

penu'c p. (mex. pano) pasar $\mathrm{S}$

epenu'c ce BRUJA pasó una bruja $\mathrm{S}$ 
$n e b a^{\prime}$ penu'c ce BRUJA ogüe'l aquí pasó una bruja anoche $\mathrm{S}$

quem t'penu'c na ate'n ¿cómo pasaste el río? S

pechu' imp. (mex. pechoa) apretar Mr S

xipechu' ¡apriétalo! S

tixpechu' japriétame! S

(bec?) nobe'c el mío Fr Jo

$m o b e^{\prime} c$ el tuyo Fr Jo

$i b e^{\prime} c$ el suyo $\mathrm{P}$

peque' imp.; pec p.; peque'z f. (mex. paca)

lavar Jo M MrS

xapeque $e^{\prime}$ na $a^{\prime} z t$ ¡limpia la olla! S

xicapeque noROPA ¡lava $\mathrm{mi}$ ropa! Jo

encape'c ya lo lavé $\mathrm{S}$

yac tapequetu' se fué a lavar $\mathrm{S}$

nui' ntapeque'z quago' voy a lavarlo

mañana $M$

pelu' imp.; pelu'c p. (mex. paloa) lamer S

xapelu' napa'zt ¡lame la olla! S

encapelu'c lo lamé S

$b i a^{\prime}, b a$ (mex. pia) tener A F Fr Jo M Mr P S ncobia' nixtu'n mil tengo una milpa muy pequeña $\mathrm{S}$

az ncobia' tumi'n no tengo dinero $\mathrm{P}$

tibia' nub'luga'm ¿tienes hijos? Mr S

quizco'm tibia' mob'lu' ¿cuántos hijos tienes? Mr S

te cobia' ¿qué tiene? $\mathrm{P}$

tue $^{\prime} n$ tibialu't nosotros lo tenemos $\mathrm{S}$

tilanqui't ncoba' tengo gallinas $\mathrm{S}$

$n c o b a^{\prime}$ nayo' $m$ tengo cuatro S

$n u c o b a^{\prime}$ tal $a c ̧ o^{\prime} c$ notzo'c mi traje tiene mucho lodo A

quizco'm mocha'n tiba' ¿cuántas casas tienes? S

$o m e^{\prime} m$ coba $a^{\prime}$ JOAQUINA Joaquina tiene $\operatorname{dos} \mathrm{S}$

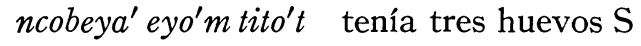

pib'lu' imp.; pib'lu'c p. (mex. pipiloa) envolver $\mathrm{S}$

xopib'lu' ca payu' ¡envuélvete en tu rebozo! S

enopib'lu'c ya me cubrí S

xiub'lu' icue'lgalo!

(pima'), nopima' mi hermana F $\mathrm{M}$ Mr

(nobima Apolonio Rosario) pina' (mex. pineua ?) hace frio F Jo M P S nopina' tengo frio F Jo M P S

pinaua' (mex. pinaua) tener vergüenza $\mathrm{S}$

pizc p. (mex. pitza en opitzaloc BIEN NACIDO)

F M S; solamente en la afrenta

PUTA motzapi'zc una puta te parió; LALMA motzapi'zc el alma te parió

pixt nube (?) $\mathrm{P}$

pitze' imp. soplar S

xipitze' na tet ¡sopla el fuego! S

picho'm (?)

picho't viejo A

pig'li' imp.; pig'le'c p.; pig'le'z f. (mex. piqui ?)

golpear

az tichapig'li' ino me golpea! A

xapig'li' ipégale! $\mathrm{P}$

capig'le'c iye' su madre le golpeó $\mathrm{S}$

etapig'le'c golpeó S

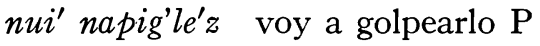

nui' ntzapig'le'z voy a golpearte $\mathrm{S}$

pict (mex. piqui) tamal A S

boz (?) echar Mr S

nuibo'z motolu' echaré atole Mr S

(bu ?) (nobu', mi hermano, Apolonio Rosario)

po imp.; potu'c ger. (mex. tlapoa) contar S xipo' motuminu' ¡cuenta tu dinero! S tapotu'c está contando S

ntapotu'c notuminu' estoy contando $\mathrm{mi}$ dinero $\mathrm{S}$

pue imp.; puc p. (cf. mex. poui) limpiar S xipue' na conebo'l ¡limpia la criatura! S encopu'c lo limpié $\mathrm{S}$

pot (mex. petlatl) petate S. Véase b'tet mopo't tu petate

puçone'l (mex. poçonilotl) espuma S

poxque'z f. (mex. pixca) pizcar $\mathrm{S}$

nui' ntapoxque'z noznu' voy a pizcar mi mazorca S

pochu't (mex. pochotl) Bombax ceiba S

polu'c p. (mex. poloa) perder Fr Jo P S

epolu'c se perdió Fr Jo

noche' polu'c todos se perdieron $\mathrm{S}$

$e m p o l u^{\prime} c$ otca' $n$ perdí el camino $\mathrm{S}$

b'tet (mex. petlatl) petate Mr S. Véase bot

nob'te't mi petate $\mathrm{S}$

ptzec (mex. pitzauac) delgado S 
(b'l) (mex. pilli) hijo F M Mr P S

$i b^{\prime} l u^{\prime}$ su hijo $\mathrm{P}$

nob'lu' mi hijo F M Mr (noblu Apolonio

Rosario)

nob'luga'm mis hijos $\mathrm{Mr} \mathrm{S}$

nob'ltzi'n tu ahijado $\mathrm{S}$

$m a, m e$ allí, ése F Fr I Jo M Mr PS ( $m a$ allá,

Apolonio Rosario)

ma ntzaci' allí voy a encontrarte $\mathrm{S}$

ma teque't ma pen LOMA ese hombre allí en la loma Jo

me que't allí está F Jo M (megue Apolonio Rosario)

me $u i^{\prime} t z$ allí viene Jo

me onque't allí hay $\mathrm{P}$

me que't ito'c $a p a^{\prime} z t$ está allí en la olla F M ma que't oque'lc está adentro S

ma imp. dar, tomar F Fr Jo M MrS. Véase meca

xima' ome'm tito't ipásame dos huevos! $\mathrm{Mr}$ xima' noxa'zt ¡toma mi jícara! S

xima' ce quagu't jtoma un palo! $\mathrm{F}$

$m a$ (?); mac p. tomar M Mr S

motzuma' te cogió $\mathrm{S}$

nichuma'c yect me cogió la lluvia M Mr S

tal cmac noxo'i, me ca nozc la tierra cogió mi pié, allí caí $\mathrm{S}$

mai (mex. maitl) mano F M $\mathrm{Mr} \mathrm{Pa}$ P S

(may Apolonio Rosario)

nomai' mi mano F M Mr S

imai' nomo't mano de mi metate F M

(noma'l, mi brazo, Apolonio Rosario)

(mateesu once, Apolonio Rosario)

(matu diez, Apolonio Rosario)

(matu eyem doce, [evidentemente trece] Apolonio Rosario)

macui'l (mex. macuilli), cinco Ep Mr P S

(maguel Apolonio Rosario)

$m a m a^{\prime} c$ p. (mex. mama) cargar S

me timama'c lo cargaste $\mathrm{S}$

(manli reir, Apolonio Rosario)

(ximanli imp. Apolonio Rosario)

malague'u F Mr melegu' Fr Jo M (mex. ma-

lacatl) huso, malacate

nomeleg $u^{\prime}$ mi malacate $\mathrm{F}$

meti' (mex. mati) saber F Jo M Mr S nen nocece' meti' IDIOMA noat'be'u yo sólo conozco el idioma de mi pueblo $\mathrm{S}$

az cmeti' noch no sé todo $\mathrm{S}$

$a z$ meti $^{\prime}$ no sé F Jo M

az nolmeti' $\operatorname{Uaxe}^{\prime} c$ no, conozco el camino para Oaxaca S (véase p. 24)

ixmeti' (mex. iximati $<$ ixtli + mati)

conocer

az nquixmeti' no los conozco S

az nchixmeti' no te conozco S (equivocación en vez de ntzixmeti' ?)

meça't (mex. maçatl) venado F Fr Jo M P S mezt (mex. metztli) luna F M (mest Apolonio Rosario)

mexti'; mexti'c p.; mexti'z f. (mex. machtia) enseñar $\mathrm{S}$

acmozmexti' ¿quién te enseña? $\mathrm{S}$

nixmexti'c noye $e^{\prime}$ me enseñó mi madre $\mathrm{S}$

$n u i^{\prime} n m e x t i{ }^{\prime} z$ voy a enseñar $\mathrm{S}$

metze' imp.; metze'z f. sentarse F I Jo Mr S

(esmeu Apolonio Rosario)

$x_{\text {metze }}^{\prime}$ isiéntate! F I Jo Mr S

leca' PARA metze ${ }^{\prime} z$ ito'c jvente a sentar

adentro! S

meche't, machete, $\mathrm{Mr} \mathrm{S}$

nomeche'u mi machete Fr Jo M Mr P S

meca' pr.; meque' imp.; mec p. (mex. maca)

dar A Ep F I Jo M Mr P S

enola'c ntzimequetu' ce RECUERDO vine a darte un recuerdo $\mathrm{S}$

$m u e^{\prime} n$ tite'z ac timeca' tú verás a quien se lo das $\mathrm{S}$

tiume' $n$ ntzimeca' dentro de un rato te daré una cosa I

az ximeque' na conebo'l ique no lo des a la criatura! S

ximeque $e^{\prime}$ CONSEJo jaconséjalo! Ep P (ximegui Apolonio Rosario)

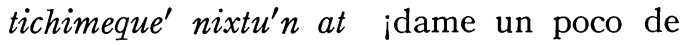
agua! $\mathrm{S}$

tichimeque $e^{\prime}$ noxamu' ¡dame mi tortilla! S tichimeque' enquibo'z ¡dame que beba!

$\mathrm{F} \mathrm{M}$

tichimeque' ce iluxa'm ¡dame una tortilla de elote! A

nichime'c el me dió S 
quizco'm time'c ¿cuántos has dado? $\mathrm{S}$ nichimequi't me dieron $\mathrm{S}$ motzimequi't te dieron $\mathrm{S}$ ticholmeque' na notupi'l ipásame mi tenate! Jo

ticholmeque' noexque't, nantzaue'z ipásame mi algodón! voy a hilar M Mr S

ticholmeque' ipásame! Fr Jo M Mr P S mie'c (mex. miac) bastante $\mathrm{S}$

mie'c motzimequi't te dieron bastante $\mathrm{S}$ micilu't (mex. miztli) puma S micho'm (mex. michin) pescado M Mr P S mixco' $m$ nixtamal

micui'x (mex. metl) maguey $\mathrm{P} \mathrm{S}$ mil (mex. milli) campo, milpa Mr P S

momi'l tu milpa $\mathrm{P} \mathrm{S}$

milyu' ce un real $\mathrm{Mr}$

(mo ?) ximoti' at jtrae agua! Mr S

moyu't (mex. moyutl) mosca

mot (mex. metlatl) metate F Fr M Mr P nomo't mi metate $\mathrm{Mr}$

(motudis, bailar, Apolonio Rosario) $m u e^{\prime} n$, tú Ep F Fr Jo M Mr P S (muen Apolonio Rosario)

$m u e^{\prime} n$ titéz tú verás $\mathrm{S}$ $\operatorname{cocho}^{\prime} m u e^{\prime} n$ ¡duerme! F M $m u e^{\prime} n$ tui' tú te vas $\mathrm{S}$

(ta) mote' imp.; mote'c p.; mote'z f. (mex. motla) tirar M Mr S xtamote' na tot ¡tira la piedra! S xtamote' na noxt ¡tira las cenizas! $\mathrm{M} \mathrm{Mr}$ enctamote'c ya lo tiré $\mathrm{S}$

QUE etitamote'c na tot ¿tiraste la piedra? S nui' nctamote'z voy a tirarlo S

moc (mex. miqui) morir F M Mr P S (moctis

Apolonio Rosario; véase mocti)

emo'c nob'lu' está muerto mi hijo F M

noch moqui't todos murieron $\mathrm{S}$

emo'c noche' todos murieron $\mathrm{S}$ mocti' imp.; mocti'c p. (mex. mictia) matar

F.M Mr S (mochis Apolonio Rosario) ximocti' ce tila'n ¡mata una gallina! Mr S ximocti' ¡mátalo! S

nixmocti'c me mató $\mathrm{S}$

moctiqui't meça't mataron venados $\mathrm{S}$

ecmoctiqui't ya lo mataron $\mathrm{S}$ (moca') tamoca' noguai' me duele la cabeza S tamoca' note' $n$ me duele la boca M Mr S (mougui, estar en pié, Apolonio Rosario)

(mo)hue' imp.; mohue'c p; mohue'z f. bañar, lavar A F I Jo M P S

mohue'; ¡lávate! F M mohue momai' ¡lávate la mano! F M enmohue'c nen ya me bañé A nen mohue'z voy a lavarme I Jo nui' mohue'z voy a bañar A F M P mamui' imp., mamui'c p. bañar $\mathrm{S}$ $x m a m u i^{\prime}$ ¡baña! S yac mamuitu' se fué a bañar $\mathrm{S}$ toque'lc mamui'c adentro se bañó! S molu'nc p. (mex. moloni) hervir S emolu'nc ya hirvió $\mathrm{S}$ moluntu'c está hirviendo S

tayua'; tayue'c p. (mex. tlayoa) obscurecer $\mathrm{P}$ a̧̧o'c tayua' está muy obscuro Mr S etayue'c ya está obscuro $\mathrm{S}$

tatenli' imp.; tatenli'z f. (mex. tlàtlani) preguntar $\mathrm{S}$

xtatenli' ipregúntale! S

nui' ntatenli'z voy a preguntarlo S

tati $^{\prime}$ imp.; tate $^{\prime} c$ p. (mex. tlatia) quemar $\mathrm{MrS}$ xtati' na oco't iquema el ocote! S

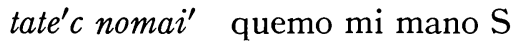

(dasupua [mex. tlaçotli + poa ?, estimar] mentir, Apolonio Rosario)

(taxpan-) tachapane' imp. (mex. tlachpana) barrer Jo M Mr S

xtachapane' na moxt ¡barre las cenizas! M Mr S

taxpana'zt escoba Mr S

notaxpana'zt tu escoba J.o

(taxiquetuc [mex. ixica], gotear, Apolonio Rosario)

(taxtoc, robar, Apolonio Rosario)

tatzi'; tatzo'c p. (mex. $t z \grave{t} t z i$ ?) ladrar S gritar $\mathrm{M} \mathrm{MrS}$

tatzi' ladra $\mathrm{S}$

etatzo'c gritó $\mathrm{M}$ Mr S (taxoc Apolonio

Rosario)

tatzotu'c tecolote está gritando el tecolote $\mathrm{S}$ tacho'm (mex. techichi ?) perro A F M P S (tachom Apolonio Rosario) 
$a$ şo' $^{\prime}$ tacho'm muchos perros $\mathrm{S}$

notachu' $\mathrm{mi}$ perro $\mathrm{P} \mathrm{S}$

taxpo'l perrito $\mathrm{S}$

tacane'l (mex. tlàcà) medio día Fr Jo S

taquechu'c p. (mex. tlacachiua) parir S etaquechu'c ya parió

tacui'c p. (mex. coa ?) pagar $\mathrm{S}$

enixtacui'c ya me pagó $\mathrm{S}$

enola'c tixtacuetu' vine a que me pagues $\mathrm{S}$ tacpa'c, sobre (mex. tlacpac). Véase ipac

(tagmum [mex. tlacomoni ?] trueno, Apolonio

Rosario)

tal (mex. tlalli) tierra Jo M Mr P S, mugre S

(tals Apolonio Rosario)

(tal) motaltzi'n tu padrino S (mex. tàtzintli) totoltzi'n el cura (nuestro padrino?)

tali' imp.; tali'c p.; tali'zf. (mex.tlalia) ponerS xtali motzo'c iponte tu camisa! S enctali'c notzo'c me puse mi traje $\mathrm{S}$ te az tinqui' motzo'c PA titali'z ¿no quieres tu traje para ponértelo? S

te (mex. tle) ¿qué? A I M Mr P S cosa

te nuca' ¿qué dices? F I M

te nacona' ¿qué hay ahora? A

te titemoa' ¿qué buscas? M Mr S

te tichua' mue'n ¿qué haces? $\mathrm{P}$

te cobia' ¿qué tienes? A

te tichimeca' ic tui' PA matbe'u ¿qué me vas a dar cuando te vayas a tu país? $\mathrm{S}$

te se usa en cuestiones, como "que" en castellano:

te tinqui at ¿que quieres agua? S

te tibia' CALENTURA ¿tienes calentura? S te tibia' mogüelu', ic tinamocti' ¿ya tienes

esposa? ¿cuándo vas á casarte? $\mathrm{Mr}$

teca' (mex. tleica) ¿por qué? F M P S

teca' tochuca' ¿por qué lloras? S

te'ipo lagarto, caimán $\mathrm{Mr} \mathrm{S}$

teyu'l (mex. tlayolli) maiz A F M Mr. P S

(tegul Apolonio Rosario)

teote $^{\prime} c$ (mex. teutlac) tarde P. Véase etiote'c $^{\prime}$

teue' (mex. tlaueltia) enojarse $\mathrm{P}$

$a_{c ̧} o^{\prime} c$ nteue' nen mucho me enojo $\mathrm{P}$

tepu' imp.; tepo'c p. (mex. tlapoa) abrir, des-

tapar P S

xotepu' jabre! $\mathrm{P}$ xitepu' ¡abre! S

ncotepo'c lo destapé S

tepo'xt (mex. tlapechtli) cama Fr Jo M Mr S

notepo'xt mi cama Jo

tepoxpo'l tabanco A

temi' imp.; temi'c, temc p. (mex. tlamia) acabar

$\mathrm{P} \mathrm{S}$

nenctemi' voy a beber $\mathrm{S}$

xtemi' ¡bebe! S

etemi'c lo acabó $\mathrm{S}$

ete' $m c$ se acabó $\mathrm{S}$

enctemi'c lo acabé P S

temoa' (mex. temoa) buscar F M Mr S

te titemoa' ¿qué buscas? F M S

nen ntemoa' nomeche' $u$ busco mi machete

M S

xtemuti ¡anda, búscalo! S

moztemutu'c andan buscándote $\mathrm{S}$

tet (mex. tletl) fuego El F Fr Jo M Mr S

(nantitulguid Apolonio Rosario)

tetoa' (mex. tlàtoa) hablar (?) $\mathrm{S}$

yut tetoa' brama el viento $\mathrm{S}$

ten (mex. tentli) boca F M $\mathrm{Mr} P \mathrm{~Pa} \mathrm{~S}$

(modenx tu boca, Apolonio Rosario)

noten mi boca F $\mathrm{M}$. Véase tzon

teque imp.; teque'c p.; teque'z f. (mex. teca) acostarse $\mathrm{S}$

$x m o t e q u e^{\prime}$ ic na quagu't jacuéstate en el

banco! $\mathrm{S}$

enmoteque'c me acosté $\mathrm{S}$

$n u i^{\prime}$ moteque'z voy a acostarme S

teque't (mex. tlacatl) hombre A F Jo Mr

P S (tequet Apolonio Rosario)

tequetque't (plural) $\mathrm{P}$

tetequetque't (plural) $\mathrm{P}$

Véase taquechu'c < teque't + chua

(tecu') (mex. tecuitli, señor) padre F M Mr PS

notecu' mi padre F M

itecu' su padre $\mathrm{P}$

tecu' imp.; tecu'z f. (mex. tlèco) subir M MrS xtecu' ¡sube! M Mr S

$n u i^{\prime}$ ntecu'z voy a subir S

tecolo't tecolote

telu'z f. (mex. tlaloa) correr S

$n u i^{\prime}$ motelu'z voy a correr S 
tiope'n (mex. teopantli) iglesia El M Mr P S (tioben Apolonio Rosario)

tiome' $n$ ahorita, despues I S tiome' $n$ tolazqui't ahorita vendremos I S

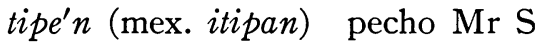
notipe'n mi pecho Mr S (nodevin Apolonio Rosario)

(tit) (mex. atetl) testículos M Mr S motitu' tus testículos M Mr S

tito't huevo $\mathrm{Mr} \mathrm{S}$. Véase tot, piedra ome'm tito't dos huevos $\mathrm{Mr}$

tice't (mex. tiçatl) huesos quemados que se usan para blanquear algodón Jo $\mathrm{S}$ tiquani' (mex. taquani) tigre $\mathrm{P} \mathrm{S}$ (tequam Apolonio Rosario)

tico' $n$ ¿cuándo? S

tico'n monaço'c ¿cuándo vino? S

tico'n tia'c ¿cuándo te fuiste? S

til (mex. tlilli) tizne S

tila'n (mex. totolin ?) gallina A Es F Jo M

$\mathrm{Mr} \mathrm{P} \mathrm{Pa} \mathrm{S}$

tilanqui't nocoba' tengo gallinas $\mathrm{S}$

notilanu' mi gallina Jo

tue'n (mex. tehuan) nosotros Es F M S (tuen

Apolonio Rosario)

tuen tibielu't tenemos $\mathrm{S}$

tup culo F M S

$i t u^{\prime} p$ su culo F M

motu'p tu culo S

motupozta'c quet está en tu culo

tupi'l (mex. topilli) tenate Jo S

notupi'l mi tenate Jo

toma'; tome' imp.; tomc p.; tome'z f. (mex. toma) desatar M Mr S

nctatoma' voy a desatarlo $\mathrm{S}$

xtatome' ¡desátalo! $\mathrm{M} \mathrm{Mr}$

enctato'mc lo desaté $\mathrm{M} \mathrm{Mr} \mathrm{S}$

nui' nctatome'z voy a desatarlo S

tome't (mex. tomatl) jitomate F M Mr S

tumi'n (mex. tomin) dinero El F I Jo M P S notumi'n mi dinero $\mathrm{El}$

notuminu' ${ }^{\prime}$ mi dinero $\mathrm{S}$

tot (mex. tetl) piedra El F M Mr P S (toot

Apolonio Rosario)

tito't huevo

chilto't piedra para moler chile $\mathrm{Mr}$ totoini' blando P S

totomo'xt (mex. totomochtli) mazorca A S

tutu't (mex. tototl, pajaro) carne F M S.

Véase neque't

totoqui' imp. (mex. toquia) atizar $\mathrm{S}$

xtotoqui' ¡atízalo! S

tutuca' (mex. totoca') pronto F Fr Jo $\mathrm{M} \mathrm{Mr}$ P S

tutuca' leca' ¡vente pronto! Fr Jo

xtutuque' na tacho'm icorre el perro! $\mathrm{M}$

toto'l $^{\prime}$ (mex. totoli' $n$ ) guajalote F M (todolem

Apolonio Rosario)

totoli't iguana verde $\mathrm{H} \mathrm{MrS}$

(to)toltzi' $n$ cura A El Fr Jo Mr P S (togolim

Apolonio Rosario). Véase taltzin

tune'l (mex. tonalli) sol F Fr Jo M P (dunel,

dia, tunel, sol, Apolonio Rosario)

tutune' calentura A

aço'c tuni' muy caliente S

tuni' quet na eso está caliente Fr Jo (tuni

Apolonio Rosario)

tinqui' mas tuni' ¿lo quieres más caliente? S xtutune' na xam icalienta la tortilla! S

toço' imp.; toço'z f. (mex. teci) moler Jo M MrS xtoço' ¡muélelo! S

nyan toço'z voy a moler Jo

nen ntoço' $z$ voy a moler $\mathrm{M} \mathrm{MrS}$

toxt (mex. textli) masa Jo M Mr S

noto'xt mi masa Jo

eque't toxt ya está (molida) la masa $\mathrm{S}$

$t u^{\prime} c h i$ pequeño, no bastante $\mathrm{F}$ M S (tu'qui

P) (tuche Apolonio Rosario)

quet tu'chi $\operatorname{nocha}^{\prime} n$ mi casa es muy pequeña $\mathrm{F}$

(tog, dios Apolonio Rosario)

-toc (mex. tic) en, adentro F M P S

$i o^{\prime} c$ at en el agua $\mathrm{S}$

ma quet ito'c apa'zt está en la olla F M

toque'lc (mex. itoc calco) en la casa $\mathrm{S}$

$x m e t z e^{\prime} i o^{\prime} c$ siéntate adentro! S

tuque'c p.; tuque'z f. (mex. toca) sembrar $\mathrm{M}$

$\mathrm{MrS}$

quizco'm etituque'c teyu'l ¿cuánto maiz has sembrado? M

eyo' $m$ oxca'zt encotuque'c sembré tres jícaras $\mathrm{M}$ 


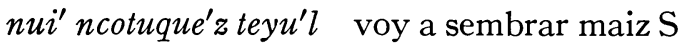
tituque'z teyu'l vas a sembrar maiz $\mathrm{Mr}$ cotuque'z él va a sembrar S

(tocdoz [mex. tequiti] trabajar, Apolonio

Rosario). Vease tqui

$n c o b a^{\prime}$ toco't tengo trabajo S

d'potz (mex. teputztli) espalda $\mathrm{S}$

nod'po'tz mi espalda S

mod'pozta'c tus espaldas S

$i d$ 'pozta'c la cara exterior de una olla $\mathrm{S}$

d'mu imp.; d'muc p.; d'muz f. (mex. temo)

bajar $\mathrm{S}$

xod' $m u^{\prime}$ ¡vente abajo! S

enod'mu'c bajé $\mathrm{S}$

nod'mu'z bajaré $\mathrm{S}$

tqui (mex. tequiti) llevar M Mr S. Véase tocdoz

nen ncotqui lo llevé M Mr S

ncotqui' lo llevó S

xitco' ¡llévalo! S

$n a$ (mex. in) el A F Fr Jo M Mr P S

ximocti' na tila'n ¡mata la gallina! S

$x_{i m a}^{\prime}$ na conebo'l itoma la criatura! F M

xtati' na oco't iquema el ocote! Mr S

chuca' na cone't llora el niño F M

unti' na conebo'l está borracho el muchacho

$\mathrm{P}$

coba' DOLOR na g'lazt la mujer tiene dolor S nocho' $x a m a^{\prime} n c$ napa'zt se quebraron todas

las ollas S

na g'lazt uzti' la mujer está embarazada S na teque't $u i^{\prime}$ unti' el hombre anda bo-

rracho $\mathrm{S}$

tuni' quet na ése está caliente Fr Jo

na mue'n tibia' tú lo tienes $\mathrm{P}$

nayo'm (mex. naui') cuatro Ep Fr Jo $\mathrm{Mr}$

P S (tayo'm A) (nayom Apolonio Rosario)

name'l éste A S (namel, aquél, Apolonio

Rosario)

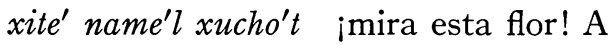

ina' o name'l aquél o éste $\mathrm{S}$

namig'li' imp. (mex. namaquiltia) vender $\mathrm{S}$

tixnamig'li' pict ¡véndeme tamales! S

namocti'; namocti'c p.; namocti'z f. (mex.

namiquia) casarse Mr P S

ic tinamocti ¿ ¿cuándo te casas? S az monamocti ¿no te casas? S

enamocti'c ya se casó $\mathrm{S}$

encnamocti'c ya me casé $\mathrm{S}$

nui' namocti'z voy a casarme $\mathrm{P}$

nan (mex. nantli, madre) S. Véase ye

monantzi'n tu madrina $\mathrm{S}$

nac (mex. nauac) cerca $\mathrm{S}$

$x$ moteque $i x n a^{\prime} c$ quagu't acuéstate frente

al banco $\mathrm{S}$

nocha'n quet ina'c ate' $n$ mi casa está en la orilla del río $\mathrm{S}$

$n a c o^{\prime}$ ahorita A F I M P S (naco', hoy, ac nacona' ¿quién? Apolonio Rosario)

naco' quet uli'c ya está bueno $\mathrm{F} \mathrm{M}$

te naco' na tui'tz ¿qué traes? A

nagua'l (mex. nahuatl) nombre del idioma de

Pochutla I

$n e b a^{\prime}$ (mex. nepa) aquí A Ep F I Jo M S (neva

Apolonio Rosario). Véase quin

leca' neba' ¡vente acá! Ep Jo

$a$ ço $^{\prime}$ c tacho'm unyo'c neba' hay aquí muchos

perros $\mathrm{S}$

neba' pec aquí entró $\mathrm{S}$

neba' quet aquí está $\mathrm{F} \mathrm{M}$

nen (mex. nèhuatl) yo $\mathrm{F}$ Jo $\mathrm{M}$ Mr S (nen

Apolonio Rosario)

az nui' nen no me voy $\mathrm{F} M$

nen az nui' no me voy $\mathrm{F} \mathrm{M} \mathrm{MrS}$

nen az nconqui' cocho'z no quiero dormir $\mathrm{F}$

$\mathrm{M}$

nen ca igüe'n tacoztu'c estoy platicando con él $\mathrm{S}$

nenepi'l (mex. nenepilli) lengua F M Mr S nonenepi'l mi lengua F M (monenevil, tu lengua, Apolonio Rosario)

neque't (mex. nacatl) carne A S (nequet,

Apolonio Rosario). Véase $t u t u ' t$

neque'zt (mex. nacaztli) oreja $\mathrm{M} \mathrm{Mr} \mathrm{P} \mathrm{Pa} \mathrm{S}$ noneque'zt mi oreja, mi oido $\mathrm{Mr} \mathrm{S}$ (mon-

gues, tu oido, Apolonio Rosario)

ni si S

nime'n (mex. nimen) ahorita I Mr P S

$n a c o^{\prime} n i m e^{\prime} n$ nui'tz ahorita vengo $\mathrm{P}$

$n a c o^{\prime} n i m e^{\prime} n n u i^{\prime}$ ahorita me voy

nintega' nada A F M P S

nintega' az nconqui' no quiero nada $\mathrm{P}$ 
ayogo' nintega' no hay nada $\mathrm{P}$ $n i x t u^{\prime} n$ (mex. -tontli) un poquito $\mathrm{F} \mathrm{M} \mathrm{MrS}$ $n i x t u^{\prime} n$ quete ${ }^{\prime}$ queda un poquito $\mathrm{Mr} \mathrm{S}$ $n i x t u^{\prime} n$ ncoba' tengo un poquito $\mathrm{S}$ $n i x t u^{\prime} n$ at nichimequi't me dieron un poquito de agua $\mathrm{S}$

nobe'c el mio F Fr Jo M

nobegu'c mañana F M (?). Véase quago' noxt (mex. nextli) ceniza, polvo El M Mr S nutza' (mex. notza) llamar S

te tinutza' noibe' ¿le llamas a mi hermana? S nocho' (mex. mochi, nochi) todo F M S nocho' tui $^{\prime}$ todos nosotros vamos F M emo'c nocho' todos murieron F M az ncalamqui' noch no me acuerdo de todo $\mathrm{S}$

noch ma til todo allí está (lleno de) tizne S nuса'; nuque'c p.; nuqueya' impf.; nuque'z f. decir, pensar F I M P S

te nuca' ¿qué dice? F I M S

ac nuca' ¿quién dice eso? $\mathrm{F} \mathrm{M}$

qui na nuqueya' así decía $\mathrm{P}$

nen nuquéc nconquiya' ce tumi'n creí que quería un peso $\mathrm{P}$

$m u e^{\prime} n$ nuque'z tu dirás

nqui; ncoya' impf.; ncoz f. (mex. nequi) querer El F Fr Jo M Mr P S

nconqui' taqua'z quiero comer F $\mathrm{M}$ az tinqui' cocho'z ¿no quieres dormir? F M az conqui' igüe' $n$ él no quiere F M az conqui'tue'n no queremos F M nconcoya' quería $\mathrm{P}$

te tinconcoya' ¿qué querías? $\mathrm{S}$

tinco'z tu querrás $\mathrm{M}$

çalu' imp. (mex. çaliui) comprar S xiçalu' ¡cómpralo! S $c e$ (mex. ce) uno A El Ep F M Mr P S (se Apolonio Rosario) ce milyu' un peso $\mathrm{Mr}$ techimeque' ce quagu't ¡dame un palo! A xte ce cue't ¡mira una culebra! A nen nocece $e^{\prime}$ meti' LA IDIOMA yo sólo conozco el idioma $\mathrm{S}$

campe' (mex. ceppa) otra vez $\mathrm{S}$ xicobe çampe $^{\prime}$ ¡hazlo otra vez! S ceque't (mex. çacatl) zacate $\mathrm{S}$ cel noce'l mi pene $\mathrm{P}$

cyeui'; cye'uc (mex. ciaui) cansado S nocyeui' estoy cansado $\mathrm{S}$ enocye'c me cansé $\mathrm{S}$ ecye'uc nod'po'tz está cansada mi espalda $\mathrm{S}$ (semibuel, veinte, Apolonio Rosario)

çumpe'l magüi'l (mex. cempualli); cemengüi'l

$\mathrm{El}$; sumpa'n magüi'l A veinticinco Fr Jo

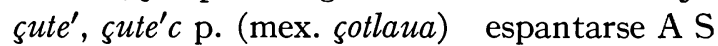
nosute'c me espanté A S

nmoçute' tengo miedo $\mathrm{S}$

f̧on (mex. centli) mazorca $\mathrm{S}$

noznu' mi mazorca $\mathrm{S}$

(sousongui, amar, Apolonio Rosario)

(z'li) (mex. celic) tierno P S

$x a m a^{\prime} n c$ p.; $x a m a n i ' z$ f. (mex. xamania) que-

brar $\mathrm{S}$

nocho' xama'nc todos se quebraron $\mathrm{S}$

exama'nc napa'zt se quebró la olla $\mathrm{S}$

$n u i^{\prime} x a m a n i ' z$ voy á quebrarlo $\mathrm{S}$

xamt (mex. xamitl, adobe) tortilla A El Es

F Fr Jo M Mr S

noxa' $m \mathrm{El}$, noxamu' Jo S mi tortilla

eluxa'mt tortilla de elote $\mathrm{A}$

xabó (castellano) jabón $\mathrm{Mr} \mathrm{P}$

$x i p u^{\prime} n$ (mex. xipintli) prepucio

$x i x e^{\prime} z$ f. (mex. $x i x a$ ) mear $\mathrm{P}$ nui' noxixe'z voy a mear $\mathrm{P}$

xict (mex. xictli) ombligo $\mathrm{S}$

$i x i^{\prime} c$ su ombligo $\mathrm{S}$

noxi'c $\mathrm{mi}$ ombligo $\mathrm{S}$

(xo-) (mex. $x o t l)$ pié A F M Mr Pa P S (xoy, Apolonio Rosario)

noxo'i mi pié $\mathrm{S}$

$x u i^{\prime}$ verde $\mathrm{M}$ Mr S. Véase $x u t$

quet $x u i^{\prime}$ na quaxilu't está verde el plátano $\mathrm{S}$ xut (mex. xiuitl) hoja M Mr S (xut, Apolonio

Rosario)

xucho't (mex. xochitl) flor A Fr Jo S

tzaue'z f. (mex. tzaua) hilar Jo M MrS nentzaue'z voy a hilar Jo $\mathrm{M} \mathrm{Mr}$ S $n u i^{\prime} n t z a u e^{\prime} z$ voy a hilar $\mathrm{M} \mathrm{Mr} \mathrm{S}$ (tzeue', ceue' ?) apagar S xitzeue' na tet japaga el fuego! S xiceue' na tequagu't japaga los tizones! S enceu' lo apagué $\mathrm{S}$ 
tzepo't (mex. tzapotl) zapote S

tzecue' imp.; tzec p.; tzecuo'z f. (mex. tzaqua) cerrar $\mathrm{M} \mathrm{MrS}$

xitzecue' na xamt ¡tapa la tortilla! M Mr S etze'c está cerrado $\mathrm{S}$

enctze'c lo cerré $\mathrm{S}$

te titze'c ¿lo cerraste? S

etze'c noneque'z está cerrado mi oido S

nui' nctzecuo'z voy a taparlo S

tzinaca' (mex. tzinacan) murcielago (chinaca,

Apolonio Rosario)

$\left(t z i n t u^{\prime} c\right.$ ) (mex. tzinti) nacer, animales y plantas $\mathrm{S}$

$z^{\prime} l i$ titzintu'c ternito está naciendo $\mathrm{S}$

titzintu'c mopollito está naciendo tu po-

llito S

tzique't (mex. tzicatl) hormiga S

(tzilini) (mex. tzilini) sonar S

tzilintu'c está sonando S

tzupine'; tzupini'c p. (mex. tzupinia) picar S nentzupine' ca ce quagu't picó con un palo $\mathrm{S}$ nixtzupini'c ce culu't me picó un alacrán S nixtzupini'c nomai' picó mi mano $\mathrm{S}$

tzupilu't (mex. tzòpilotl) zopilote. Véase cuzt (tzupcu -?) tzucua'; tzucua'c, tzupa'c (sic) p.; tzupa'z (sic) f. cortar P S

xitzucua' na opque't icorta el hilo! S

etzucua'c ya está cortado S

tzupa'c nomai'. cortó mi mano P S

PA $t z u p a^{\prime} z$ na quagu't para cortar el palo S $($ tzoma $)$; tzome'z f. (mex. tzoma) coser $\mathrm{M} \mathrm{Mr}$ $\mathrm{S}$

titzome'z tu vas a coser $\mathrm{S}$

$n u i^{\prime}$ ntatzome'z voy a coser $\mathrm{S}$

ntatzontu'c estaba cosiendo $\mathrm{S}$

tzon (mex. tzontli) pelo $\mathrm{Mr} \mathrm{P} \mathrm{Pa} \mathrm{S}$ (sont,

Apolonio Rosario)

$m o t z o ' n$ tu pelo $\mathrm{P}$

motentzo' $n$ tu barba $\mathrm{P}$ (tu boca-pelo)

(modensen Apolonio Rosario)

tzoct paño, traje, camisa M Mr S

notzo'c mi traje

tzocua'zt (mex. tzicauaztli) peine $\mathrm{S}$

$n t z o c u i ' z$ voy a peinar $\mathrm{S}$

tzulu' imp.; tzulu'c p. (mex. tzoloa) sacudir M

$\mathrm{MrS}$ xitzulu' na tepo'xt sacudí la cama M Mr S entzulu'c lo sacudí $\mathrm{S}$

(chan) (mex. chantli) casa A El F Fr M Mr

$\mathrm{P} \mathrm{Pa} \mathrm{S}$ (nochan, mi casa Apolonio

Rosario)

huhio'm nocha'n mi casa es grande F M

$i c h a^{\prime} n$ su casa $\mathrm{S}$

quizco'm mocha'n tiba' ¿cuántas casas tienes? $\mathrm{S}$

$\operatorname{toch}^{\prime} n$ nuestra casa

che pr. é imp.; chez f. (mex. chia) esperar S neba' ntzoche' aquí te espero S

xiche' ¡espera! S

tixche' ¡espérame! S

nui' nchez voy a esperar S

$n t z o c h e t u^{\prime} c$ estoy esperándote $\mathrm{S}$

$($ chibilu') (mex. tepilli ?) vulva $\mathrm{P}$

mochibilu' tu vulva $\mathrm{P}$

chicala't corriente del río $\mathrm{P}$

chique'c (mex. chicauac) duro P S

chil (mex. chilli) chile Mr S (chil, Apolonio

Rosario)

chilto't piedra para moler chile $\mathrm{Mr}$

chua': pr.; chue' imp.; chuc p. (mex. chiua)

hacer A El F Fr I taquechuc Jo M Mr P S

nixchua' PULGA me pica (hace) la pulga $\mathrm{S}$ te tichua' ¿qué haces? F M

chua' DAÑo quaxilu't $x u i^{\prime}$ hace daño el plátano verde $\mathrm{S}$

PA chue' AMARRAR para amarrarlo $\mathrm{M} \mathrm{Mr}$ xichue' ¡haz! A El F Fr I Jo M Mr P S echu'c uli'c lo hizo bueno F M

tichulu't vamos a hacerlo $\mathrm{S}$

chutu'c PLANCHANDO está planchando S

entzuchu'c VENDER yo te lo he vendido A.

Véase taquechu'c

chupe'c (mex. chipauac) blanco A M Mr S

choch (mex. chica, chicha) escupir, saliva S

$i c h o^{\prime}$ ch su saliva $\mathrm{S}$

nocho'ch mi saliva $\mathrm{S}$

chucha'c huele A Fr Jo P S

chuca' (mex. choca) llorar F M P S

chuca' na cone't llora el niño F M

teca' chucá ¿por qué llora? F M

teca' tochuca' ¿por qué lloras? S 
nchuca', a'mba emo'c nob'lu' lloro, porque se murió mi hijo $\mathrm{S}$

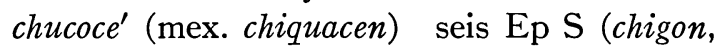
Apolonio Rosario)

chucula't (mex. chocolatl) chocolate F M MrS (chol ?) nocholu' mi hermano Fr Jo S mocholuga'm tus hermanos $\mathrm{S}$

$c a$ (mex. ca) (particula) A F M Mr ca $x u i^{\prime}$ janda! F M ca $x u i \mathrm{PA} m o c h a^{\prime} n$ jvete a tu casa! $\mathrm{Mr}$ ca quixui' PA nocha'n iándale a tu casa! Mr ca (mex.can) dónde A F I M Mr P S ca tui' ¿por dónde te vas? M Mr S ca tiba' at ¿a dónde tienes agua? $\mathrm{S}$ ca tyac ¿por dónde fuiste? S campa' (mex. campa) ¿dónde? A El P POR campa'tui' ¿por dónde te vas? A $c a$ (mex. $c a)$ con F M P S nen ca igüe' $n$ natacoztu'c estoy platicando con él S

nen tzupine' ca ce quagu't picó con un palo $\mathrm{S}$

ca nen conmigo $\mathrm{P}$

ximocti' ca moxo'i ¡mátalo con tu pié! S (cayivima, frio, Apolonio Rosario) cayu' caballo Fr Jo P S (cayu, Apolonio Rosario)

cayupo'l potro, caballito $\mathrm{S}$

cau (mex. caua) quedarse $\mathrm{S}$

nen mocau' nocece' me quedo sólo $\mathrm{S}$ az cauanqui' no sirve F M P (az caban$\left.q u i^{\prime} ?\right)$

caxani'; caxa'nc p. (mex.caxani) sanar M Mr $\mathrm{S}$

te motzeti'c DOCTOR te caxani' ¿te dijo el doctor que sane? $\mathrm{M}$ caxani' está sanando $\mathrm{S}$ yulicyuli'c ui caxa'nz despacio va a sanar $\mathrm{M}$

calamqui'; calamco'z f. acordarse S az ncalamqui' noch no me acuerdo de todo $\mathrm{S}$

nui' ncalamco'z voy a acordarme S queue' imp.; queue'z f. (mex. cauia) guardar $\mathrm{M} \operatorname{MrS}$ (aqueue'?)

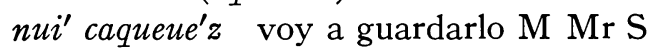

xaqueue iguárdalo! S

quem (mex. quen) cómo $\mathrm{S}$

quem tpenu'c na ate' $n$ ¿cómo pasaste el río?S quet; quetya' (mex. catqui) hay A Ep F Fr I

Jo M Mr P S

neba' quet aquí está $\mathrm{F} \mathrm{M}$

ma quet allí está F M

az quet uli'c no está bueno Ep

quet $t u^{\prime} c h i$ nocha' $n$ mi casa es pequeña $\mathrm{F} M$ ma quet oque'lc está allí adentro F M

ma quet ite'nc apa'zt está debajo de la olla S na quet ma está allí I

neba' quetya' aquí estaba $\mathrm{P} \mathrm{S}$

onque't (mex. onca) hay $\mathrm{P} \mathrm{S}$

$a \varsigma^{\prime} c$ onque't cue't hay muchas culebras $\mathrm{P}$

quago' az nonque't neba' mañana no estaré aquí $\mathrm{S}$

eyonque't xamt hay tortillas Fr Jo

quexque'mt (mex. quechquemitl) huipil F Jo

$\mathrm{M} \mathrm{Pa}$ (quext, coxt pescueso)

noquexque'm mi huipil Jo

quequi'; quec p.; quez f. (mex. caqui) oir F M

$\mathrm{MrS}$

az ncoquequi' no lo oigo $\mathrm{Mr} \mathrm{S}$

toquequi' oyes F M

encoque'c oí S

$n u i^{\prime} n c o q u e^{\prime} z$ voy a oir S

(quel) (mex. calli) casa F M

ito'c quelc en la casa F M

quin (mex. quin) solamente con neba' y na A Jo P S

quineba' nui' acá me voy Jo

leca' PA quineba' ivente acá! A

quina' nuqueya' así decía A $\mathrm{P}$

quiça'; quice' imp.; quizc p.; quice'z f. (mex. quiça) salir F I M P S

tiquiça' mato'lc sales fuera $\mathrm{S}$

xiquice' S; quice' F M P S ¡sal!

ma que't quiztu'c apoto'ct allí está saliendo

el humo $\mathrm{S}$

equi'zc tune'l salió el sol $\mathrm{P}$

nen quice'z saldré I

quizco'm (mex. quezqui) ¿cuántos? $\mathrm{Mr} \mathrm{S}$ quizco' $m$ meleque't tiba' ¿cuántos malacates tienes? $\mathrm{S}$ 
quixi' imp.; quixi'c p.; quixi'z f. (mex. quixtia) sacar $\mathrm{S}$

xiquixi' ¡sácalo! S

enquixi'c lo saqué $\mathrm{S}$

nquixi'z voy a sacarlo $\mathrm{S}$

co naco' ahora S P. Véase naco'

(qua); quac p.; quazf. (mex. qua) comer A F

Fr Jo M Mr P S

etaqua'c comió M P

totaqua'c has comido $\mathrm{S}$

az nconqui' taqua'z no quiero comer F M

az tiqua'z no lo comerás F M

totaqua'z comeremos A (totaguasquit, Apo-

lonio Rosario)

xicque' na quaxilu't PA tiquazqui't asa el plátano para que lo comamos $\mathrm{F}$ M S

xicueti' ce pan PA tiquazqui't compra un

pan para que lo comamos $\mathrm{S}$

PA $n q u a^{\prime} z$ para que lo coma S

xtaquati' jvete a comer! S

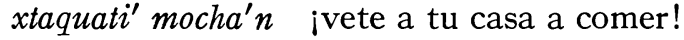

$\mathrm{S}$

xtaquaqui' ivente a comer! S

nen naquacamqui' tengo hambre Fr Jo S

noquaçonqui' tengo hambre $\mathrm{P}$

$c u a^{\prime}$; cue imp.; cuc p.; $c u a^{\prime} z$ f. (mex. coa) com-

prar F Fr Jo M Mr S

ticucua' lo compras Fr Jo

xicue ¡cómpralo! S

xicueti' jvete a comprarlo! S

encocu'c lo compré S

encucu'c teyu'l compré maiz $\mathrm{M}$ Mr S

az ticua'z tutu't pa taqua'z ¿no comprarás

carne para comerla? $\mathrm{F}$

(quait) (mex. quaitl) cabeza F M Mr P Pa S noquai' mi cabeza (noquay, Apolonio Rosario)

quane' imp. rascar $\mathrm{P} \mathrm{S}$

xaquane' ¿ráscalo! S

chua' naquantu'c está rascándose $\mathrm{S}$

quanco'ch costal Fr Jo

quaxa'xt faja M Mr S

quaxilu't (mex. coaxilotl) plátano A El F M

$\mathrm{MrS}$

quago' mañana A Fr Jo Mr P S (cuago, goago, Apolonio Rosario) quagu't (mex. quauitl) palo, árbol, leña banco, cárcel, fusil A El F Fr Jo M Mr S noquagu't mi fusil $\mathrm{El}$

tequagu't tizón $\mathrm{S}$

nyac quagutu' fuí a leñar $\mathrm{S}$

antu' quaguzqui't ¡vámonos a leñar! A Jo

$\mathrm{Mr}$

quala't lagartija S

guala'c (mex. huallauh) venir F I M P S

$n e b a^{\prime}$ guala'c acá vino $\mathrm{S}$

eguala'c totoltzi'n vino el cura $\mathrm{P}$

enola'c vine

ic tola'c ¿cuándo viniste? S

nola'c tixtagüetu' vine para que me pagues $\mathrm{S}$

tolaqui't venimos $\mathrm{S}$

ic molaqui't ¿cuándo vinieron? S

(qual ?) noqualu' mi lado $\mathrm{M} \mathrm{Mr} \mathrm{S}$

(güe, cue ?) nogüe'u mi marido $\mathrm{Mr} P \mathrm{~S}$ (noquehu, Apolonio Rosario)

(cueit) (mex. cueitl) enagua El F Jo $\mathrm{M} \mathrm{Mr}$

$\mathrm{Pa} \mathrm{S}$

nocue' $i$ mi enagua $\mathrm{Mr}$

$c u e^{\prime} t$ (mex. coatl) culebra F Fr Jo M S

(cuet, Apolonio Rosario)

$a c ̧ o^{\prime} c$ cue't muchas culebras F M

cuete'xt (mex. cuetlaxtli) cuero, piel S

güecha'l (véase mex. uiptla) pasado mañana

M Mr P S (guechal sí, Apolonio Rosario)

Güeuatla'n LAGUNA nombre antiguo de

Pochutla Fr Jo

güeque' (mex. ueca) lejos Fr Jo (asoc gueque

muy lejos, Apolonio Rosario)

(güel ?) igüelu' su esposa Fr Jo M Mr P S

te micu'l mogüelu' ¿cómo se llama tu

esposa? Mr S (noquelu, mi esposa, Apo-

lonio Rosario)

(cuizca' ?) traer I

$a c^{\prime} c$ VIENTO cuizca LA LUNA mucho viento

trajo la luna I

cuixo' $m$ iguana (mex. cuixin MILAN) F Fr Jo $\mathrm{M} \mathrm{S}$

(cuique) (mex. cuica) cantar S

ntacuiquetu'c estoy cantando S

coyu'd (mex. coyotl) coyote (Apolonio Rosario)

$\operatorname{cope}^{\prime} c$ (mex. copetic) grueso S 
coma'l (mex. comalli) comal A F Fr Jo M Mr nocomalu' mi comal A F

nocumale'u (castellano comadre) mi comadre F S

nocumpale'u (castellano compadre) mi compadre El Fr Jo Mr P S

cumt (mex. comitl) cántaro F Fr Jo M MrS cute't (mex. cuitlatl) mierda A

cone't (mex. conetl) niño El F M P

$x_{i t e}^{\prime}$ na cone't mira el niño $\mathrm{F} \mathrm{M}$

cocone't niños F M P (coconet, muchacho;

coconets, muchachas, Apolonio Rosario) conebo'l criatura F M P (conevol, Apolonio

Rosario)

coconebo'l criaturas $\mathrm{P}$

(contze ?) xucontze' ¡échalo (adentro)! Fr Jo $\mathrm{Mr} \mathrm{S}$

nenconce' $z$ voy a hacer tortillas Fr Jo

enconce'c hice tortillas Fr Jo

cuzt zopilote Mr S. Véase tzupilu't

coztu'c. Véase $(t a) c t z e$ platicar

coxt (mex. quechtli) pescuezo M Mr S. Véase

quexque'mt

moco'ch tu pescuezo $\mathrm{S}$

coxqui' (mex. quequexquia) comezón A S

nicoxqui tengo comezón $\mathrm{S}$

$\left(\operatorname{cochi}^{\prime}\right)$; $\operatorname{coxc}$ p.; $\operatorname{coch}^{\prime} z$ f. (mex. cochi) dormir

F M Mr P S

eco'xc durmió $\mathrm{S}$

cocoxtu'c está durmiendo F M

nen cocho'z voy a dormir F M (cochos,

Apolonio Rosario)

az nconqui cocho'z no quiero dormir $\mathrm{F}$

cocho' mue'n ¡duerme! F

xucochoti' jvete a dormir! S

aço'c ncocoxni' deseo mucho dormir $\mathrm{P}$

cuchi' puerco M MrS

cocoa' (mex. cocoa) enfermo F M Mr S

nococoa' nomai' tengo enferma la mano

$\mathrm{F} \mathrm{M}$

az nococoa' no estoy enfermo S

nococoa' noye' mi madre está enferma $\mathrm{S}$

cug'li; cug'lu'c p. frio M Mr S

aço'c cug'li' se enfrió mucho M Mr S

ecug'lu'c ya se enfrió $\mathrm{S}$

quet cug'luni' hace frio $\mathrm{S}$ (cul ?) (mex. colli, antepasado ?) nombre te nicu'l muén ¿cómo te llamas? F M QUE micu'l moye' ¿cómo se llama tu madre? $\mathrm{S}$ que tmocu'l ¿cómo te llamas? S icu'l mogüelu' MARIA mi esposa se llama María S

culu't (mex. colotl) alacrán A F M colme'n (mex.queman ?) hace poco tiempo $\mathrm{S}$ colme'n nola'c vino hace poco tiempo S icolme'n yac se fué hace poco tiempo $\mathrm{S}$ colme'n quizc salió hace poco tiempo $\mathrm{S}$ ctza; ctze imp.; cozc, ctzec p.; ctzez f. (mex. quetza) levantar F M S az $u e^{\prime} l$ noctza'n no se puede levantar $\mathrm{S}$ xmoctze' ipárate! F M

te $n \operatorname{coctze} e^{\prime} c$ ¿en qué pisé? $\mathrm{S}$

enmoco'zc me levanté $\mathrm{S}$

$n u i^{\prime} n \operatorname{coctze}^{\prime} z$ nocha' $n$ voy a parar a mi casa $\mathrm{S}$

az nconqui' timoctze' z no quierolevantarme F M

(ta) $c t z e^{\prime}$ (mex. quetza) platicar F M S

totactze' hablas F M

xtactze' ¡habla! S

tacoztu'c está platicando F M S (dacus, tagustuc, Apolonio Rosario)

totacoztu'c estamos platicando S, estás platicando $\mathrm{S}$

$n t a c o z t u^{\prime} c$ neba' estoy platicando aquí $\mathrm{S}$ g'lazt (mex. quilaztli) mujer A F Fr Jo M Mr P S (claxtl, Apolonio Rosario) quig'lazqui't mujeres $\mathrm{P} \mathrm{S}$ omeme't quig'lazqui't dos mujeres $\mathrm{P}$ nog'la'zt mi esposa Fr Jo g'lazpo'l muchacha A

(ta) g'lua' (mex. cuiloa) escribir S tag'lutu'c está escribiendo $\mathrm{S}$ xtag'luti' ¡escribe! S

huhio'm (mex. uei) grande F Fr Jo M Mr S $h_{u l u}$ imp.; hulu'c p. (mex. oilia) pepenar, ${ }^{1}$ recoger, separar $\mathrm{S}$ xihulu' na teyu'l ipepena el maiz S!

1 Véase Cecilio A. Robelo, Dicionario de Aztequismos (Cuernavaca, I904), p. 632. 
encuhulu'c lo pepené $\mathrm{S}$

leca' ¡vente! A Ep Fr Jo Mr P S

lipu' bule $\mathrm{F}$ Fr Jo $\mathrm{M}$ Mr calabazo con cintura $^{1}$ (mex. ilpia atar ?)

(lyu) (mex. [no] yollo) corazón Mr S (noliu, Apolonio Rosario)

aço'c tamoca' nolyu' mucho me duele el corazón S

luxalyu' (castellano rosario) Fr Jo S

noluxalyu' mi rosario Fr S

\section{CASTELLANO-POCHUTLA}

abrir tepu'

acá quineba' (quin)

acabar temi'

acordarse calamqui' (nocho')

acostarse teque' $\left(i x n a^{\prime} c\right)$

adentro toc $\left(m a, m e t z e^{\prime}, q u e t\right)$. Véase EN

agua at (meca, toc, nixtu'n, ca)

aguacate aueque't

ahijado b'ltzin

ahora $n a c o^{\prime}\left(a z, u i^{\prime} t z\right)$; nacona ${ }^{\prime}$ (te); conaco'

alas $y a j a i^{\prime}$

alacrán culu't (tzupine')

algodón oxque't (meca)

allí ma (ato'lc, aci', ui', ui'tz, yect, pen, toc, nocho', quet, quiça')

amar sousongui

ancho pate'c

¡anda! ca (ui)

andar agueneumi

anoche ogüe'l (apeco', pen, penu'c)

antier icualgua' (igualgua' ?)

apagar tzeue', ceue (?)

apestar iye'c

apretar pech $u^{\prime}$

aquí $n e b a^{\prime}\left(a y a g o^{\prime}, a p e c o^{\prime}, a c i^{\prime}, a c ̧ o^{\prime} c\right.$, penu'c, guala'c, quet)

árbol quagu't

arco escocu'l

asar ixque' (qua)

así qui na (quin)

atar $i l p i^{\prime}$

atizar totoqui'

atole etu'l (boz)

ayer algua'

ayudar petebi' (ac)

bailar motudi's

bajar d'mu

banco quagu't (ixna'c, teque')

1 Véase Cecilio A. Robelo, Dicionario de Aztequismos, Apendice, p. 2.

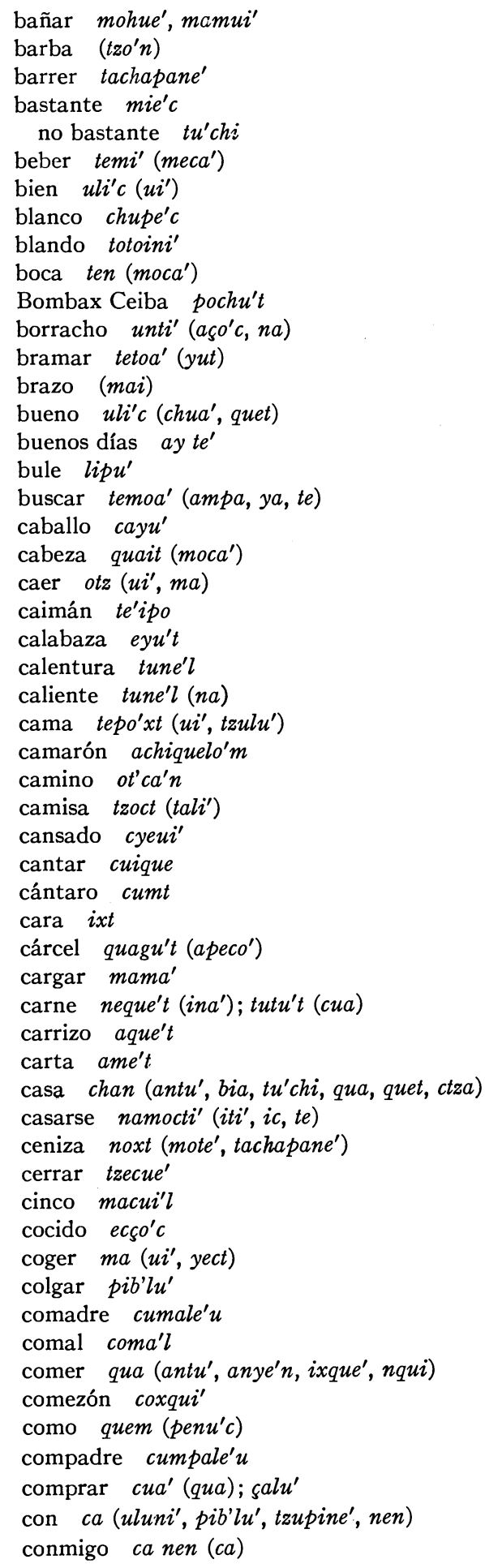




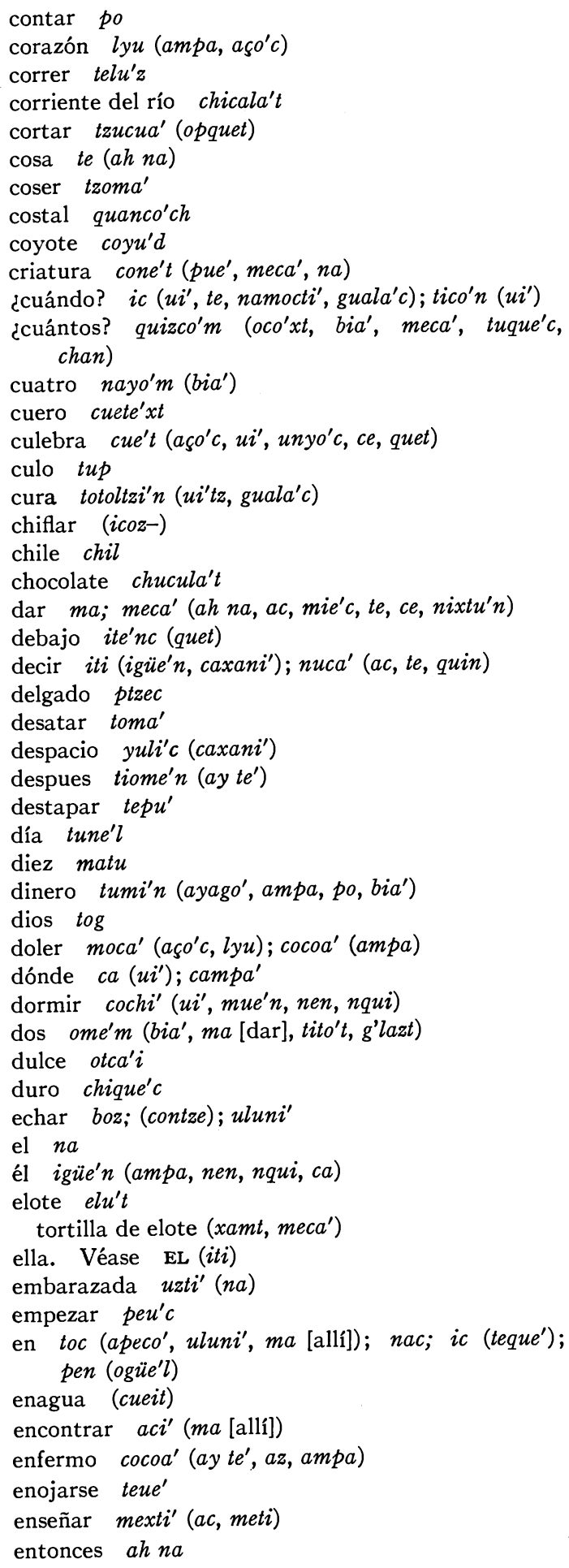

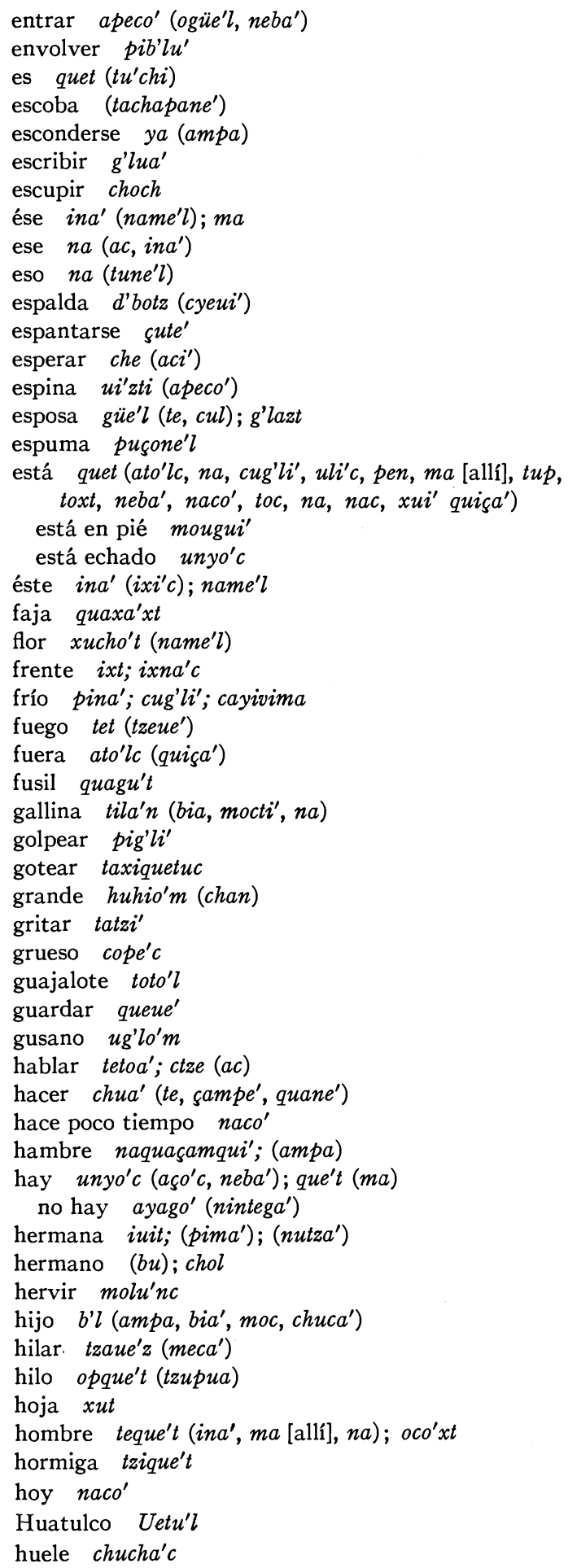


hueso ot

huesos quemados tice't

huevo tito't (ome'm, bia', ma [dar], tot)

huipil quexque'mt

humo apoto'ct (quice')

huso malage'u (quizco'm)

iglesia $\operatorname{tiope}^{\prime} n$ (pen)

iguana cuixo'm

iguana verde totoli't

ir $u i^{\prime}$ (ay te', ampa, ate'n)

jabón $x a b o^{\prime}$

jicalpézte pebe't

jícara oxca'zt (ma [dar], tuque'c)

lado qual

ladrar tatzi'

lagarto $t e^{\prime} i p o$

lagartija quala't

lamer pelu'

lavar peque' (yulicyuli'c)

lavar mohue'

lejos güeque'

lengua nenepi'l

leña quagu't

levantar tqui; ctza (ue'l)

limpiar pue'

lo $m e$ (mama'c)

lodo tal (bia)

luna mezt

llamar nutza'

llevar tqui (eti')

llorar chuca' (ampa,az, te, na)

lluvia yect ( $u i^{\prime}, u i^{\prime} t z, m a$ [tomar])

machete meche't (temoa')

madre ye (ayago', iti, pig'li', meti', cul, cocoa')

madrina nan

maduro eç̧o'c

maguey micui'x

maiz teyu'l (az, ui', tuque'c, cua)

malacate malague'u (quizco'm)

mano mai (tati', tzupine', cocoa')

mañana quago' $\left(a z, u i^{\prime}, u i^{\prime} t z\right.$, peque $\left.e^{\prime}, q u e t\right)$; nobegu'c mañana, pasado güecha'l

mar at

marido güe' (ampa)

masa toxt

matar mocti' (ca [con], na)

mazorca totomo'xt; çon (poxce'z)

mear $x i x e^{\prime} z$

medio día tacane'l

menear uluni'

mentir dasupua

metate mot (mai)

mierda cute't

milpa mil $\left(b i a^{\prime}, i t a^{\prime}\right)$

mio nobe'c mirar ita' (ampa, az, name'l, ce, cone't)

mojar $a u e^{\prime} c$

moler toço' $\left(u i^{\prime}\right)$

morir moc (ampa, nocho', chuca)

mosca moyu't

mover uluni'

muchacha g'lazt

muchacho cone't $\left(i n a^{\prime}, i x i^{\prime} c, n a\right)$

mucho $a o^{\prime} c$ (aue c, ampa, unyo'c, ug'li', bia', teue', tacho'm, neba', cochi', cuizca', cue't, quet)

mugre tal

mujer g'lazt (na)

murciélago tzinaca'

muy $a c ̧ o^{\prime} c$ (etiote'c, eueta'c, ina', tayua', tune'l, lyu)

nacer $t z i n t u^{\prime} c$

nada nintega' (ayago')

Nahuatl nagua'l

nariz yeque't

niño cone't (az, entzute'c, ita', na, chuca')

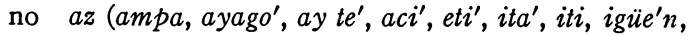
$u i^{\prime}, u e^{\prime} l, u i^{\prime} t z, u l u n i^{\prime}, b i a^{\prime}, p^{\prime}{ }^{\prime} l i^{\prime}$, meti', meca', tali', cau, namocti', nqui, nen, nintega', nocho' calamqui', quet, quequi', qua, cua', cochi', cocoa', ctza); ayago'; ay te' (az)

noche ogüel (apeco')

nombre cul (güel)

nosotros $t u e^{\prime} n$ (bia', nqui)

nube pixt (?)

Oaxaca $\operatorname{Uaxe}^{\prime} c($ anye' $n$, meti')

ocote oco't (na)

oido neque'zt (tzecue)

oir quequi'

ojo ixtotolu't

olla apa'zt (ite'nc, yuli'c, uluni', ma [allí peque', pelu', toc, na, xama'nc, quet)

olvidar yactangui

ombligo xict

once mateesu

oreja neque'zt

orita (ahorita) naco'; nime'n; (aci', ui'tz); tiume'n $\left(m e c a^{\prime}\right)$

obscurecer tayua'

otra vez çampe'

padre $\left(t e c u^{\prime}\right)\left(i n a^{\prime}, i x i^{\prime} c, u i^{\prime}\right)$

padrino (tal)

pagar tagüi'c (guala'c)

palo quagu't (uluni', pen, ma [dar], tzupine', ca [con], ce)

pan pan

paño payo'; tzoct $\left(\right.$ ig'ti' $^{\prime}$ )

papel $a m e^{\prime} t$

parecido $i x i^{\prime} c\left(i n a^{\prime}\right)$

parir taquechu'c; pizc

pasar penu'c (quem)

pasearse paxalu- 


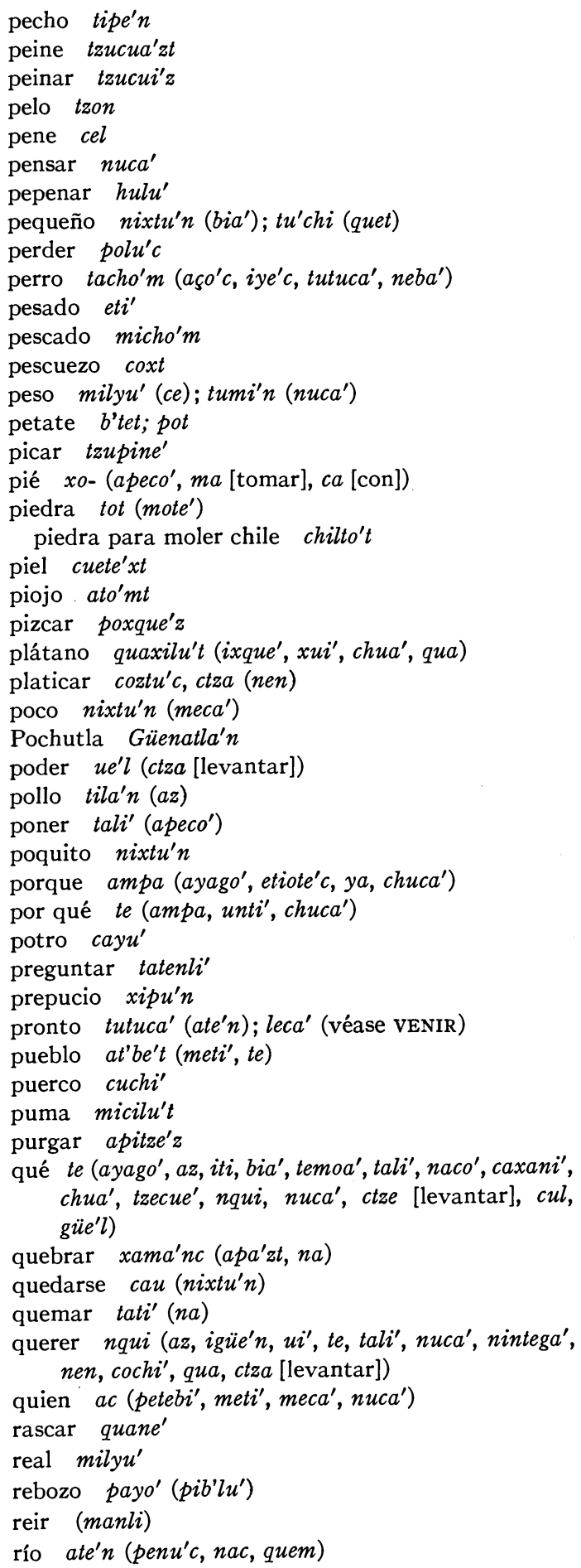

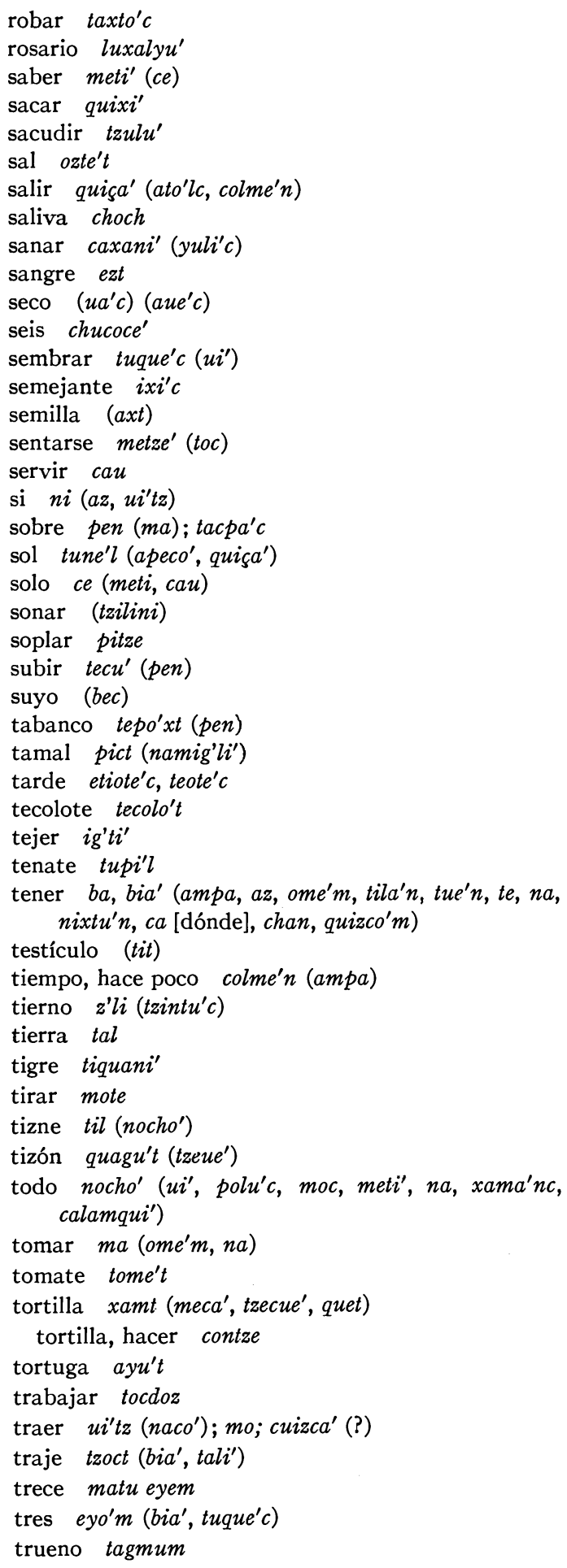


tu $\quad m u e^{\prime} n$ (ampa, ac, unti', meca', te, na, nuca, cochi', $\left.i l p i^{\prime}, c u l\right)$

uno ce (apeco', az, ig'ti', uluni', penu'c, ma [tomar], meca', mocti', nuca', tzupine', ca [con], qua)

uña oxt

¡vámonos! antu' (ate'n, quagu't)

veinte semibuel

veinticinco çumpe'l magüi'l; cemengüi'l; çumpa'n magüi'l

venado meça't (mocti')

vender namig'li'

venir $u i^{\prime} t z$ (az, yect, ma [allí], nime'n); guala'c (algua', ic, tiome'n, meca', colme'n); leca' (antu', metze', neba', tutuca', quin). Véase PRONTo ver $i t a^{\prime}\left(a m p a, a z, m e c a^{\prime}, m u e^{\prime} n\right)$

verde $x u i^{\prime}$ (chua)

vergüenza, tener pinaua'

viejo picho't $\left(\right.$ ina $\left.^{\prime}\right)$; eueta'c

viento $y u t$

volar pata'nc $\left(u i^{\prime}\right)$

vomitar entzute'c

vulva chibilu'

yo nen (ampa, eti', icoz-, ui', mohue, meti', ita', toço', teue, temoa', tqui, nuca', ca [con], cau, ce, qua, cochi', quiça')

zapote $t z e p o^{\prime} t$

zopilote $t z u p i l u^{\prime} t, c u z t$ 